e-Journal of Educational

Research, Assessment and Evaluation

\section{REIIEVE}

Revista ELectrónica de Investigación y EValuación Educativa

\title{
ACTITUDES Y VELOCIDAD EN JÓVENES. APLICACIÓN DE UN PROGRAMA DE EDUCACIÓN VIAL
} [Attitude and speed in young people. Implementation of a program of
driver education]

\begin{abstract}
Article record
About authors

$\underline{\text { HTML format }}$
\end{abstract}

Abstract
Contemporary Theories of change of attitudes defends that
these depends on the way to think, to act and to feel of the
people. If we touch upon each one of these three main
components related between we can influence in the pre-
disposition to act. The application of a program of speed
self-control to ample of young drivers, using a design pre-
test-postest with control group and using a Factor Analysis
shows that is possible the change of attitudes.
Keywords
Change of attitudes; Speed; Investigation in road educa-
tion and security; Road education and formation; Road
security.

por

\author{
Jariot Garcia, Mercè (Merce.Jariot@uab.cat) \\ Montané Capdevila, Josep (Josep.Montane@uab.cat)
}

\section{Ficha del artículo \\ $\underline{\text { Sobre los autores }}$ \\ Formato HTML}

\section{Introducción}

El concepto de actitud ha sufrido una considerable evolución desde que Znaniecki en 1918 la definió como un estado de pensamiento que predispone a que un individuo acepte un objeto en Edwin (2003). Fazöö (1986) la reducía a los procesos y a la estructura de la memoria. Thurstone (1931) la relacionó con el pensamiento y las emociones. Fishbein (1963) incluyó la creencia como un elemento cognitivo y la relación con un objeto.

Resumen
Las teorías contemporáneas del cambio de actitudes de-
fienden que éstas dependen de la manera de pensar, actuar
y sentir de las personas. Si se incide en cada uno de estos
tres factores íntimamente relacionados entre si podemos
influir en la predisposición a actuar. La aplicación de un
programa de autocontrol de velocidad a una muestra de
preconductores, utilizando un diseño pretest-postest con
grupo control y utilizando un análisis factorial pone de
manifiesto que es posible el cambio de actitudes en los
jóvenes.
Descriptores
Cambio de actitudes; Velocidad; Investigación en educa-
ción y seguridad vial; Educación y formación viales; Se-
guridad vial.

El hecho de añadir a las actitudes las cualidades de las emociones permite que se consideren como un proceso de aceptación y rechazo o de componente positivo o negativo, como por ejemplo la tendencia a la seguridad o al riesgo Montané, Jariot y Rodríguez (2007)

Allport (1954) consideró el factor conductual como el tercer componente que condiciona la actitud. En esta misma línea Wicker (1969) defiende que en la búsqueda de los componentes de las actitudes hay que intro- 
ducir aspectos de comportamiento verbal y de respuestas abiertas. La actitud entendida de una forma triádica: ideas, emociones y conductas explican la predisposición de una persona a actuar de una forma determinada. Esta afirmación la defienden también, Ajzen y Fishbein (1980), la revisión realizada por la OECD (1994) y Montané, Jariot y Rodríguez (2007).

Erwin (2003), Montané, Jariot y Rodríguez, (2007) y la recopilación realizada por Albarracín, Jonson y Zanna (2005) nos permiten entender la actitud como la predisposición a actuar de una manera determinada y dicha predisposición se debe a la incidencia de los componentes cognitivos, afectivos y conductuales de manera interactiva.

Los trabajos de Ajzen y Fisbein (1980, 2000 y 2005) nos introducen en aspectos que relacionan entre si los tres componentes que configuran la actitud desde la perspectiva de la persuasión. Los componentes cognitivos, la conducta y las emociones no solo son los tres elementos que predisponen a actuar de manera determinada sino que cada uno influye también en los otros dos restantes.

Por una parte las ideas influyen en la ejecución de la conducta y ésta expresada de manera repetitiva favorece la creación de hábitos de comportamiento fáciles de expresar, pero por otra, las emociones, la expresión de la asertividad y de manera especial de la persuasión, influyen en la aceptación de los aspectos cognitivos. De manera especial las aportaciones de Erwin (2005) y Bohner (2005) abren un nuevo camino al relacionar la persuasión en la forma de modificar las ideas y los comportamientos. Las emociones favorables o desfavorables al comportamiento deseado actúan también como reforzadores positivos o negativos respectivamente favoreciendo la predisposición de la conducta a modo de condicionamiento.

Tal como se muestra a continuación los tres factores que configuran la actitud a saber: pensamiento, comportamientos y emo- ciones Montané, Jariot y Rodríguez (2007), aparecen de manera progresiva en el tiempo. En la actualidad las teorías contemporáneas del cambio de actitudes, contemplan la relación de estos tres factores entre si y con el mismo objeto de la actitud: la predisposición a actuar de una manera determinada. Es en este espacio, donde se ubica la persuasión como un elemento que impregna el proceso cognitivo y emotivo. También la manera de comportarse influye en los otros dos componentes e incluso con la misma actitud. Los procesos de intervención y de cambio de actitudes se relacionan con la intervención en cada uno de estos factores entendidos como un proceso interactivo. Veamos como ha ido evolucionando

\subsection{La manera de pensar influye en la actitud}

La primera aportación en la clasificación de factores presentes en la actitud, la contempla como un factor que depende en exclusividad del conocimiento. Osgoog y Tannenbaum (1955); Festinger (1957); Abelson y Rosenberg (1958); Heider (1958); McGuire (1964); Feldman (1966) y Cooper (1977).

Esta primera aproximación al cambio de actitudes es muy reduccionista puesto que contempla casi exclusivamente factores con predominio lógico o cognitivo, sin tener en cuenta factores afectivos y conductuales que intervienen de manera preponderante en el cambio de actitudes.

Todos los estudios anteriormente citados muestran que los resultados obtenidos con la teoría de la disonancia cognitiva son discutibles puesto que parece que intervienen otras variables, entre las que destacan las de defensa del yo, de valores o expectativas personales. De esta manera Kelman (1953), Festinger y Carlsmith (1959), Hoyt (1972) y Wicklund y Brem (1976) explican que se produce la disonancia cuando esta presente la responsabilidad personal. 
En una etapa posterior, autores como Abelson (1983), Aronson (1992) y Olson y Stone (2005) relacionan los componentes cognitivos con los emotivos o Stone y Cooper (2001) que desarrollan temas socio - cognitivos donde la reducción de la disonancia nos introduce en contextos sociales, en nuestro caso relacionados con la creencia y la convicción para reducir los accidentes de tráfico.

Por último, Beauvois y Joule (1996) nos indica como se puede explicar el cambio de actitud desde la radicalidad, es decir cuando todos los elementos cognitivos presentan su máximo valor junto con los factores de mantenimiento de la consistencia. Un ejemplo aplicado a la reducción de la accidentalidad seria la denominada "tolerancia cero al alcohol”, sin embargo este concepto parece que dista mucho del modelo de cambio de actitudes que se propone en esta investigación.

\subsection{La segunda aportación en la clasifi-} cación de factores presentes en la actitud, incluye los elementos cognitivos y algunos aspectos relacionados con la emoción.

Las aportaciones de las teorías de la homeostasis, la toma de decisiones, de la compensación del riesgo, de la percepción del riesgo y la educación emocional presentan algunos aspectos que superan la simple cognición como factor determinante de la actitud.

La teoría de la homeostasis se ha aplicado a diferentes ámbitos como el consumo de tabaco, substancias tóxicas, riesgos laborales y a los accidentes de tráfico. En el tema de prevención de accidentes de tráfico la intervención desde esta teoría tendería a mantener los resultados en el intervalo óptimo: evitando los dos extremos considerados de riesgo (Wilde, 1994, 1998; Peltzman, 1975; Evans, 1985).

La homeostasis del riesgo se puede considerar como el riesgo en proceso de evolución, normalmente moderado o si se prefiere un proceso de salud estable dentro de una franja de salud aceptable. Esta premisa hace que sea difícil adscribirse a la teoría de la homeostasis si pretende reducir el riesgo de manera óptima con tendencia a la "tolerancia cero".

La teoría de la toma de decisiones con autores como Näätanen y Summala (1976), integraron los factores de riesgo subjetivo, motivos de riesgo y seguridad, comportamientos de inhibición, riesgo sugerido, factores de personalidad, la motivación como impulso, las expectativas, los deseos de cambio, la toma de decisiones, la acción y percepción del riesgo a la conducción segura, que, a partir de su relación con la teoría de la toma de decisiones permitió elaborar un modelo de la toma de decisiones en relación con la conducción segura. Sin embargo, una de sus principales limitaciones para fomentar el cambio de actitud es que se fundamenta, de manera prioritaria en los procesos perceptivos y es excesivamente generalista.

La teoría de la compensación del riesgo se presenta como una aplicación de la teoría de la homeostasis y de la percepción del riesgo a los factores del análisis del núcleo del accidente de tráfico, en este sentido los trabajos de Ullerberg (2002) parten de la teoría de la compensación homeostática del riesgo e inciden en el factor humano como el que explica los accidentes de tráfico unificados en la figura del conductor y de sus procesos de compensación, pero apenas incluye nuevos factores como la adquisición de hábitos o la educación de las emociones, con lo que es difícil que esta teoría evolucione hacia el modelo de cambio de actitudes.

Con los trabajos de Reason (1990) y Parker, Manstead y Stradling (1995) se avanza en el conocimiento de nuevos factores de riesgo pero no en la forma de intervenir para reducir la accidentalidad.

La teoría de la percepción del riesgo es una de las teorías más aceptadas y utilizadas para reducir los comportamientos de riesgo y de los accidentes de tráfico. Dicha teoría se 
fundamenta en dos principios básicos: la percepción del riesgo. Y que altos niveles de percepción del riesgo se relacionan también con bajos niveles de comportamientos de riesgo.

Bajo estas dos premisas, la teoría de la percepción del riesgo, se limita a intervenir en los procesos perceptivos. Así Peters, Burraston y Mertz (2004) sostienen que las personas tienden a reducir el riesgo en función de los grados de dificultad (a más dificultad más riesgo).

Las aportaciones de McDaniels, Kamlet y Fisher (1992) y las de Alhakami y Slovic (2004) indican que los cambios en la percepción influyen en la mejora de los comportamientos de riesgo.

Estudios más actuales como los de Peters, Burraston y Mertz (2004) afirman que la emoción es la llave para modificar la percepción del riesgo El sentimiento incluye una situación emocional de atracción o rechazo (buena o mala) en relación a un estímulo (idea, persona u objeto) de riesgo o seguridad. Este componente emotivo se relaciona con la cognición. El discurso supone una evolución de la teoría de la percepción del riesgo versus la teoría de la actitud, no es de extrañar esta evolución ya que el discurso de estos autores se realiza en el marco de los diferentes temas descritos por el The Handbook of Attitudes, Albarracín, Jonson y Zanna (2005) en el que tiene lugar una interpretación desde la teoría de las actitudes.

Una de las limitaciones de esta teoría es que correlaciona directamente la percepción del riesgo con comportamientos de reducción del mismo como un fenómeno simple de un proceso lógico, y en este sentido los comportamientos de las personas, entre los que se encuentran los comportamientos de riesgo, no dependen solamente de los procesos del pensamiento sino de otros factores concomitantes relacionados con los comportamientos y las emociones.
La educación emocional ofrece avances relacionados con la prevención de accidentes de tráfico. La conciencia emocional positiva, controlada, con alto nivel de capacidad social para generar emociones positivas, nos permite conocer sin prejuicios los factores de riesgo y desear aplicar las medidas de prevención más convenientes (Bisquerra, 2003).

Son frecuentes los autores que relacionan los procesos de pensamiento con las emociones y su repercusión con el cambio de actitudes (Haddock y Zanna 1993 y Haddock y Maio 2004).

Las emociones favorables o desfavorables se aplican a diferentes objetos de la actitud como comportamientos altruistas tal y como afirman Brekler y Wiggins (1989), a pensar de manera determinada y al consumo de substancias adictivas como defienden Trafimow y Sheran (1998)

\subsection{La tercera aportación incluye la cognición, la conducta, las emociones y su interrelación.}

La visión actual del cambio de actitudes defiende que la tendencia a comportarse de una manera determinada depende de la manera de pensar, de comportarse y de las emociones que acompañan a la manera de pensar y de actuar (Ajzen y Fisbein, 1980; Assum, 1997; Montané, Jariot y Rodríguez, 2007).

El estudio de las interrelaciones de estos tres factores entre si abre una nueva perspectiva a la investigación. Desde esta visión se acepta que las emociones influyen en la manera de pensar y de actuar (en la configuración de los hábitos de comportamiento), también se admite que las ideas y la forma de comportarse influyen en la predisposición a actuar, pero también las actitudes influyen de nuevo y remodelan las ideas, además, los nuevos hábitos pueden incidir en nuevas formas de transformar las emociones y en definitiva este proceso interactivo y abierto, también puede modificar de nuevo las actitudes. En este proceso activo es donde se 
sitúan las bases para entender los pasos para modificar, evolucionar y fortalecer el cambio de actitudes de una manera estable. Es en esta interacción donde puede introducirse la persuasión como un nuevo elemento que matiza las teorías contemporáneas del cambio de actitudes.

\subsection{Bases para la elaboración de un modelo para el cambio de actitudes}

Todas las limitaciones anteriormente citadas de los modelos y enfoques que de una manera u otra intentan reducir la accidentalidad, nos conducen a buscar y probar un modelo que promueva cambios en los niveles de riesgo que tengan como objetivo último la “tolerancia cero" a la accidentalidad.

Cuando se aplican los tres componentes de la actitud a nuestro objeto de estudio las ideas indicarían como influye la información en la actitud, las emociones incluirían sentimientos favorables o desfavorables al riesgo y la comprensión de los componentes conductuales ayudarían a entender como está instalada la actitud y su posible resistencia facilidad de cambio.

Los tres componentes de la actitud están íntimamente relacionados. La intensidad de cada uno de ellos y su interrelación explicarían, en gran parte, la predisposición a actuar de una manera determinada. Con todo lo dicho ya se puede adivinar lo difícil que resulta cambiar las actitudes incidiendo solo en cada uno de sus componentes por separado: las ideas, las emociones o los comportamientos. El siguiente esquema pretende resumir la información más relevante del modelo de cambio de actitudes que aquí se presenta.

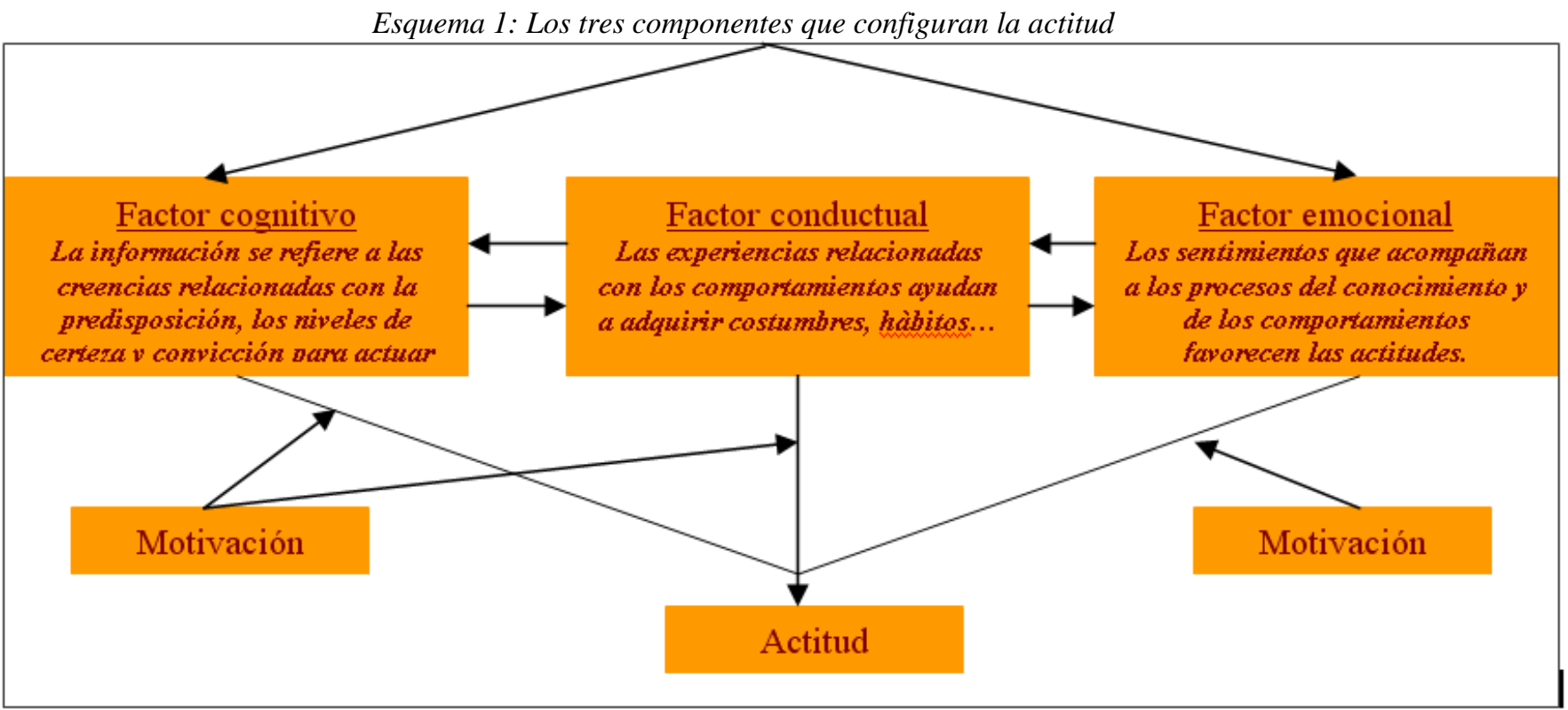

La estabilidad y predisposición al cambio de cada uno de los tres componentes de las actitudes explica hasta que punto ésta es un componente estable y susceptible al cambio.

Las teorías contemporáneas del cambio de actitudes incluyen aspectos de la persuasión en la cognición y en los elementos emotivos.
Los estudios de Ajzen y Fishbein (2005) constatan como la actitud y la conducta incluyen aspectos cognitivos como razonamiento, intención y planificación, aspectos relacionados con los hábitos y condicionamiento y de alguna forma también introduce aspectos emotivos que influyen en la previsión del comportamiento. La estructura del 
afecto descrita por el estudio de metanálisis de Armitatge y Cooner (2001) y las teorías contemporáneas de la persuasión de Erwin (2001), presentan aspectos relacionados con la planificación de la conducta y los límites del razonamiento de la acción aspectos íntimamente relacionados con las actitudes y la persuasión.

Han existido intentos de aplicar este modelo a los accidentes de tráfico, relacionando las actitudes con los accidentes para constatar la relación existente entre estas y la presencia - ausencia de riesgo. Los trabajos de Näätänen y Sumala $(1974,1976)$ consideran los componentes del factor humano en la conducción presumiendo que su mejora repercutirá en un progreso de la responsabilidad y en la adquisición de las destrezas necesarias para reducir los accidentes de trafico. Los trabajos de Montané y Ferrer (1993), Rodríguez (2001), Montané y Jariot (2003, 2004 y 2005), Montané, Jariot, Martínez, Amador y Rodríguez (2003), Montané, Martínez y Jariot (2004), Jariot y Rodríguez (2005) y Jariot y Rodríguez (2006) explicitan que para iniciar el cambio de actitudes se requiere intervenir en las dimensiones de las actitudes, en todos y cada uno de los factores de riesgo presentes durante el proceso de intervención.

\subsection{Aplicación del modelo de cambio de actitudes a la prevención de los acci- dentes de tráfico.}

La formación para el cambio de actitudes en relación con un o unos factores de riesgo, desde este modelo de cambio de actitudes, sigue los siguientes pasos:

1. Se inicia la observación del factor de riesgo para deducir como debería ser la conducción segura: en relación con como debería ser el comportamiento del conductor para reducir los accidentes de tráfico desde ese factor.

2. Definir los objetivos operativos de la conducción segura y eficaz: "Reducir el número de accidentes y la intensidad de los daños como consecuencia de estos".

3. Evaluar los comportamientos de riesgo que realiza el colectivo al que va a ir dirigido el programa de intervención.

4. Deducir las necesidades de formación que muestran los conductores y las que, consecuentemente deberían integrar desde la seguridad.

5. Constatar las teorías y modelos a utilizar en el proceso formativo del cambio de actitudes.

6. Contemplar los criterios de optimización en la elaboración de los programas de intervención.

7. Elaborar el programa para la mejora de la conducción segura que integre los tres elementos que conforman la actitud.

8. Detectar necesidades y formar a los profesionales que aplicaran los programas. 9. Aplicar el programa de formación y constatar los resultados, tomando como referencia un diseño de investigación pretest - postest con grupo control.

\subsection{El programa de educación vial pa- ra mejorar la seguridad a partir del au- tocontrol de la velocidad.}

El programa de autocontrol de la velocidad para preconductores es uno de los programas diseñados para promover el cambio de actitudes, tal como se presenta en este trabajo, consta de cinco fases:

FASE 1: Evaluación inicial: con el fin de conocer el riesgo - seguridad inicial en relación con determinados factores de riesgo y preparar la intervención de una manera personalizada. Esta fase se subdivide en dos subfases:

A.- Aplicación del cuestionario QAR Precon para conocer el perfil de riesgo de los futuros conductores y elegir el programa de cambio de actitudes que va a aplicarse.

B.- Aplicación del cuestionario especifico de velocidad para elegir aquellos elemen- 
tos del factor de riesgo en los que se debe incidir de manera más específica.

FASE 2: Mejora de la información: con el objetivo que el preconductor reflexione sobre sus creencias y se inicie el cambio, se debe trabajar de manera personalizada, por ello la información se relaciona con cada uno de los ítems que conforman el cuestionario de evaluación inicial. En el cuadro siguiente se presenta un ejemplo de cómo se diseña la información relacionada con cada ítem.

Cuadro 1: Ejemplo del diseño de la información

Ítem 3.- Si puedo, por ciudad iré a más de 50 $\mathrm{Km} / \mathrm{h}$.

Esta tercera pregunta se sigue relacionando con una apreciación inexacta del efecto del aumento de la velocidad en los daños que se derivan de un accidente. Cada incremento lineal de la velocidad comporta un incremento exponencial de ésta, que se hace muy evidente cuando la velocidad es muy alta. Aun así hay que tener en cuenta que, el crecimiento exponencial se produce también en incrementos pequeños o a bajas velocidades, de manera que el riesgo derivado de circular a 40 kilómetros por hora o incluso a 30 puede suponer, en determinadas circunstancias la diferencia entre la vida y la muerte en un atropellamiento. A 50 $\mathrm{km} / \mathrm{h}$ un golpe a un peatón puede ser fácilmente mortal....

Cuando se aplica esta fase, la información se proporciona en función de los ítems más deficitarios, de manera adaptada a las necesidades de los usuarios; a partir de las respuestas que estos han ofrecido en el cuestionario específico sobre el factor de riesgo. Por ello el formador debe interactuar con cada uno de los preconductores, ofreciendo toda aquella información que pueda incidir en la mejora de las creencias de los alumnos. Es muy importante que la información que se ofrezca sea impactante, real, actualizada, objetiva y extraída de fuentes fiables. En ningún caso el formador, durante esta fase, introducirá valores o juzgará las opiniones de los alumnos, simplemente rebatirá todas las creencias de los alumnos utilizando informaciones impactantes sobre las consecuencias de las conductas imprudentes con el factor de riesgo velocidad.
FASE 3: La revisión de hábitos o mejora de las destrezas: contempla hasta que punto el comportamiento de riesgo en el factor velocidad está instalado y que alternativas existen para transformar esos comportamientos de riesgo en costumbres, hábitos y automatismos de seguridad en relación con la velocidad.

En esta fase el profesor de formación vial debe descubrir, preguntando a los alumnos, el nivel de tendencia al riesgo y la preparación para el cambio de comportamiento. Esta fase consta de tres niveles:

1er nivel: conocer como se ha instalado ese comportamiento de riesgo en los usuarios. En esta primera fase el preconductor o preconductora habla de sus costumbres y el profesor de formación vial escucha y sigue preguntando sin entrar en discusión o debate.

$2^{\circ}$ nivel: saber y hacer consciente al usuario de hasta qué punto sabe que su comportamiento es una conducta de riesgo.

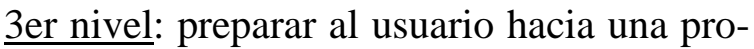
puesta de cambio de comportamiento de riesgo y convertirlo en seguridad. Los compromisos que adquiere el preconductor en esta fase son hipotéticos y muestra hasta que punto el alumno esta dispuesto a cambiar.

Por ello el profesor de formación vial deberá realizar todas las preguntas necesarias al preconductor para que verbalice sus costumbres y sea consciente de su nivel de riesgo en la conducción. En ningún caso el formador deberá abrir debate ni juzgará los comportamientos de los preconductores. Estos niveles de reflexión harán que el preconductor empiece a estar preparado para el cambio de actitud final.

Se presenta a continuación un ejemplo de las preguntas que se utilizan en esta fase para diagnosticar el nivel de tendencia al riesgo y la preparación hacia el cambio de los preconductores. 


\section{Cuadro 2: Ejemplo de preguntas de la fase de revisión de comportamientos y hábitos}

\section{Preguntas del nivel 1: Conocer hasta que punto se ha instalado el hábito de riesgo en rela-} ción con la velocidad.

¿Qué hace? ¿Cómo lo hace? ¿Con quien lo hace? ¿Qué siente cuando lo hace?

Preguntas del nivel 2: Hacer consciente al preconductor de cómo tiene instalado el hábito de riesgo:

¿Estos comportamientos los hace sin pensar? ¿Actúa de manera inconsciente?...

Preguntas del nivel 3: preparar los comportamientos que deberá practicar para transformar los hábitos de riesgo en seguridad:

¿Cuáles de estos comportamientos cree que son más peligrosos o graves? ¿Por donde podría empezar, si tuviera que cambiar, para mejorar su nivel de seguridad?

FASE 4: La transmisión o integración de sentimientos o emociones: ayudan a fortalecer las ideas con los comportamientos de seguridad. Incluye la transmisión de emociones, sentimientos y valores relacionados con la comprensión experiencial de las consecuencias de la velocidad en los accidentes. En esta fase la vivencia en primera persona de un testimonio que ha padecido las consecuencias de un accidente de tráfico es una herramienta muy importante para integrar emociones. Durante 15 - 25 minutos el profesor de formación vial presenta al testimonio y este describe el accidente y explica su vivencia sobre las consecuencias del accidente de tráfico inmediatas y a largo plazo. Al finalizar los preconductores explicitan cuales son sus compromisos para que no se produzcan situaciones como las que les ha presentado el testimonio.

FASE 5: La evaluación final: permite detectar a corto y medio plazo las ganancias obtenidas al comparar las diferencias entre la evaluación de entrada y la de salida después del desarrollo del proceso formativo. Para ello se vuelve a administrar el cuestionario de evaluación inicial pero redactado des de la perspectiva de futuro. Se tabulan e interpretan los resultados y se comentan las mejoras en seguridad, así como aquellos resultados que el profesor de formación vial crea oportunos.

\section{MÉTODO.}

Con esta investigación se pretende comprobar en qué medida el desarrollo de un programa fundamentado en el modelo de cambio de actitudes aplicado a la reducción de los accidentes de tráfico en relación con el factor de riesgo velocidad consigue cambiar las actitudes de jóvenes que van a iniciarse como conductores de turismo en relación con este factor de riesgo.

Se ha optado por utilizar un diseño de investigación simple y clásico para confirmar o rechazar nuestra hipótesis de trabajo. Se esquematiza a continuación el proceso seguido: 


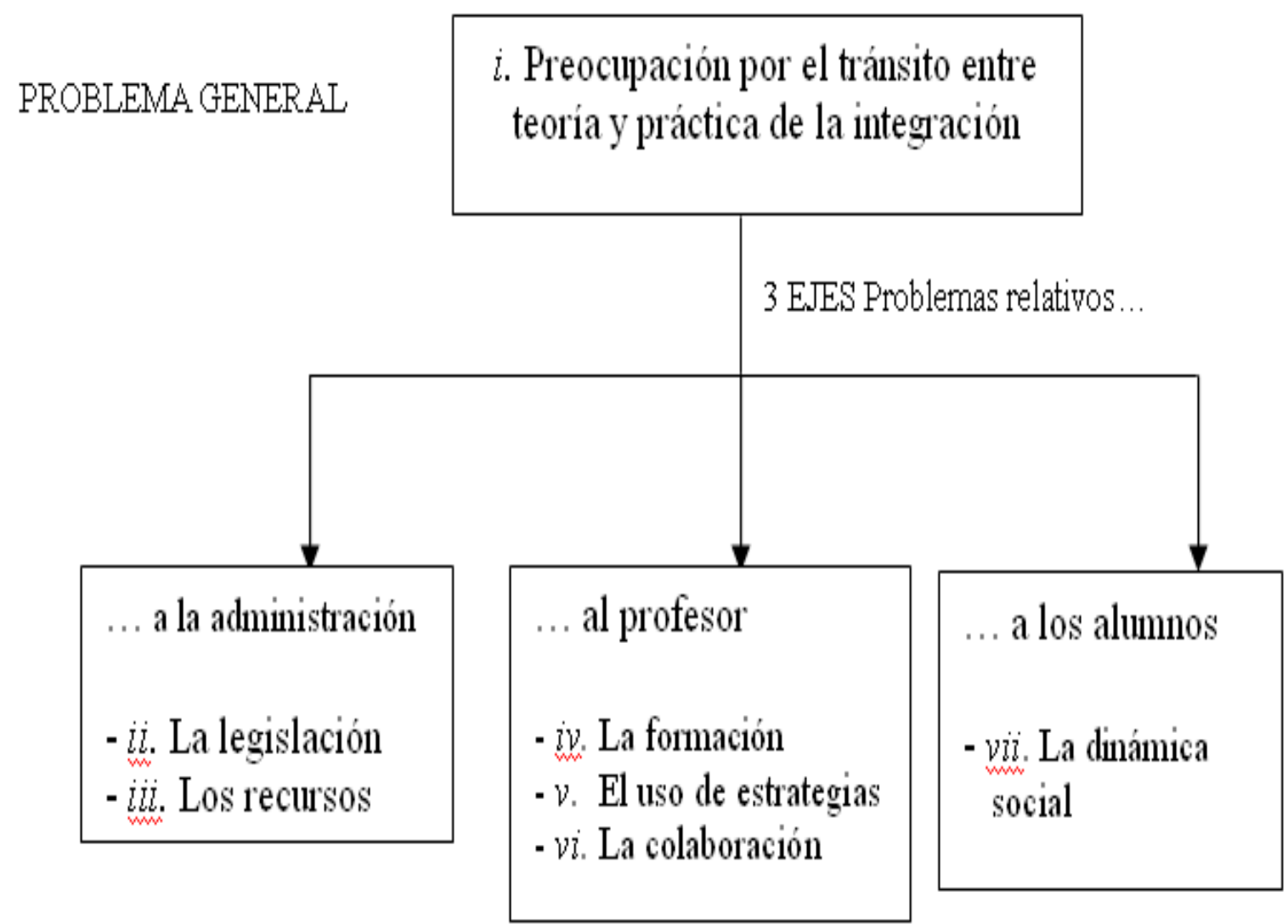

Tal y como puede observarse se trata de un diseño pretest - postest con grupo control. La prueba estadística utilizada (mediante el paquete estadístico SPSS 15.0 para Windows) ha sido un análisis de varianza con medidas repetidas, concretamente el procedimiento Modelo lineal general con medidas repetidas para estudiar el efecto del programa de cambio de actitudes relacionado con la velocidad en los jóvenes preconductores.

La temporización de la investigación viene condicionada por la duración del proceso formativo, puesto que se ha integrado en él. De este modo la aplicación del QAR - Precon (Cuestionario de evaluación del riesgo para preconductores) se realizó antes de iniciar el proceso formativo para conocer el factor de riesgo que debería trabajarse en los jóvenes. La aplicación de los cuestionarios específicos para la evaluación del nivel de riesgo en relación con la velocidad se realizó antes de iniciar el proceso formativo tradi- cional y antes de la aplicación del programa de cambio de actitudes y cuando finaliza el proceso de formación y el programa.

El grupo experimental consta de 135 jóvenes preconductores, mientras que el control está formado por 40 sujetos. La muestra proviene de las cuatro provincias catalanas.

Se han utilizado dos cuestionarios distintos que seguidamente se detallan:

$\varnothing$ QAR- Precon (Cuestionario de evaluación del riesgo para preconductores): detecta posibles indicadores de riesgo en el inicio de la conducción, nos permite encontrar los puntos débiles de cada preconductor e incidir de manera especial en todos aquellos aspectos más relacionados con la reducción de la accidentalidad. Esta dividido en tres bloques que contienen todos los objetos de evaluación. Estos bloques son:

I. Salud y cuidado personal: se trata de conocer la importancia que da cada perso- 
na a su propia integridad y salud, si estos hábitos no están logrados o no se consideran importantes será más difícil integrar hábitos de conducción segura y responsable. Formado por 6 ítems.

II. Los grandes factores de riesgo: trata de manera especifica todos los factores de riesgo que pueden afectar a la conducción: alcohol, velocidad, tendencia al riesgo, conductor, acompañante, vehiculo, vía, tráfico, climatología, normas y señales de tráfico. Formado por 75 ítems.

III. Test de madurez de la conducción eficaz: trata de valorar la propia trayectoria del alumno durante la formación y el papel que cada agente debe jugar en la prevención de la accidentalidad. Formado por 9 ítems

$\varnothing$ El cuestionario especifico de evaluación del nivel de riesgo en relación a la velocidad: su objetivo es evaluar el riesgo de manera individual y de forma más específica, puesto que sólo recoge información del factor de riesgo. Este cuestionario consta de 12 ítems que analizan conocimientos, compor- tamientos y emociones en relación con la velocidad.

\section{RESULTADOS}

\subsection{Comprobación de la fiabilidad y validez de los instrumentos de recogida de información.}

Se realizaron estudios de validez y fiabilidad del Cuestionario QAR-Precon. A continuación se presentan los resultados de aplicar la prueba Reliability del Paquete estadístico SPSS 15.0 para Windows, obteniendo un valor alpha de Cronbach de 0,9914, con lo que afirmarse que el cuestionario, de manera global, incluyendo los factores de riesgo $\mathrm{y}$ el test de madurez presenta un nivel alto y muy aceptable de fiabilidad y consistencia interna.

El nivel de consistencia interna de cada factor también es muy elevada y los ítems presentan una puntuación muy homogénea, tal y como se puede comprobar en la tabla 1:

Tabla 1: Coeficiente alfa de Cronbach por factores.

\begin{tabular}{|l|c|c|}
\hline \multicolumn{1}{|c|}{ FACTORES } & ÍTEMS & $\begin{array}{c}\text { COEFICIENTE } \\
\text { ALFA }\end{array}$ \\
\hline 1. Salud & 1 al 6 & .879 \\
\hline 2. Grandes Factores de riesgo & 7 al 27 & .864 \\
2.1. Alcohol & 7 al 14 & .923 \\
2.2. Velocidad & 15 al 20 & .892 \\
2.3. Tendencia al riesgo & 21 al 27 & .910 \\
\hline 3. Otros factores de riesgo & 28 al 81 & .987 \\
3.1. Conductor & 28 al 42 & .948 \\
3.2. Acompañante & 43 al 45 & .812 \\
3.3. Vehiculo & 46 al 58 & .958 \\
3.4. Vía & 59 al 63 & .934 \\
3.5. Tráfico & 64 al 70 & .926 \\
3.6. Clima & 71 al 77 & .922 \\
3.7. Normas y señales & 78 al 81 \\
\hline 4. Test de madurez & 82 al 90 & .891 \\
\hline
\end{tabular}

En conclusión el Cuestionario de Evaluación del Riesgo del Preconductor (QAR-Precon):

- Es un cuestionario muy fiable en el sentido que evalúa un solo factor, que según el marco teórico esta relacionado con la seguridad y el riesgo.

- Todos los ítems están íntimamente relacionados entre sí. 
- No contiene ninguna pregunta que se mueva en sus respuestas de manera arbitraria.

- Todos los factores presentan un índice elevado de consistencia interna.

- Incluso el bloque de madurez del preconductor forma un todo coherente de cuestiones relacionadas, pero también diferenciadas entre sí.

La validez externa se ha constatado relacionando el tipo de vehículo utilizado (bicicleta o ciclomotor) y frecuencia de uso por el preconductor con el número de accidentes e intensidad de daños

La validez general es altamente significativa, aunque la correlación sea relativamente baja, tanto en el número de accidentes ( $\mathrm{p}=$ 0,000 y r de Pearson $=.279$ ) como en la intensidad de los daños ( $\mathrm{p}=0,021 \mathrm{y} \mathrm{r}$ de Pearson $=$ 0.145). En definitiva, los preconductores que puntúan alto en general en el cuestionario también puntuarán alto en número de accidentes e intensidad de los daños.

Se volvió a comprobar la fiabilidad del cuestionario con la muestra de preconductores que integran esta investigación y se comprueba que: el coeficiente alpha de Cronbach es de 0.923 , con lo que se confirma que el cuestio- nario, de manera global, incluyendo los factores de riesgo y el test de madurez presenta un nivel alto y muy aceptable de fiabilidad y consistencia interna cuando se aplica a otra muestra.

La fiablilidad del cuestionario específico de evaluación del factor de riesgo velocidad se realizó utilizando nuevamente el coeficiente alpha de Cronbach y se comprobó que éste era de 0.864 , con lo que podemos afirmar que el cuestionario, de manera global tiene un nivel alto y aceptable de fiabilidad y de consistencia interna. Si analizamos las aportaciones de los ítems se observa que la mayoría de los ítems están relacionados entre sí y que presentan un índice elevado de consistencia interna. El ítem "Cuando voy de acompañante me gusta que el conductor corra" presenta un alfa sin el ítem de 0,871 , mientras que el alfa global es de 0.864. Es decir, si se elimina este ítem la fiabilidad aumenta (el ítem no aporta fiabilidad, aún más, la hace disminuir, sin embargo se decide conservarlo en la puntuación total puesto que su contenido es importante en cuanto a la valoración positiva personal que los preconductores realizan del constructo velocidad (consultar tabla 2).

Tabla 2: Resultados de la aplicación del coeficiente alpha de Cronbach: análisis de los ítems del cuestionario especifico de velocidad

\begin{tabular}{|c|c|c|}
\hline ÍTEMS & $\begin{array}{l}\text { Correlación del } \\
\text { elemento - total } \\
\text { (corregida) }\end{array}$ & $\begin{array}{l}\text { ALPHA si se } \\
\text { elimina el ele- } \\
\text { mento }\end{array}$ \\
\hline Me gusta correr & .603 & .850 \\
\hline Creo que iré a más de $15 \mathrm{Km} / \mathrm{h}$ de la velocidad señalada & .591 & .850 \\
\hline Si puedo, por ciudad, iré a más de $50 \mathrm{Km} / \mathrm{h}$ & .629 & .848 \\
\hline Creo que tengo habilidades para correr con facilidad & .589 & .850 \\
\hline Pienso que correré más en las vías conocidas & .583 & .851 \\
\hline Creo que en autopista y otras vías rápidas me será fácil correr con facilidad & .428 & .860 \\
\hline Para mi, uno de los mejores atractivos de la conducción es la velocidad & .647 & .846 \\
\hline $\begin{array}{l}\text { Pienso que los turismos actuales son para correr, por eso tienen las presta- } \\
\text { ciones que tienen }\end{array}$ & .674 & .845 \\
\hline Si aprendo a correr, me ayudará a vivir mejor & .377 & .864 \\
\hline Cuando voy de acompañante, me gusta que el conductor corra. & .273 & .871 \\
\hline Pienso que un buen conductor/a debe saber correr & .522 & .855 \\
\hline Si me esfuerzo en conducir bien podré correr más & .634 & .849 \\
\hline
\end{tabular}


Por último se comprobó la validez concurrente de la escala específica, utilizando como referente el bloque de ítems de velocidad del cuestionario QAR - Precon. La correlación de Pearson entre los dos cuestionarios es de $0,461(p=0,012)$ lo que muestra que existe una correlación estadísticamente significativa entre los dos instrumentos. Con lo que puede afirmarse que el cuestionario específico de velocidad posee validez concurrente. En definitiva, los preconductores que puntúan alto en el cuestionario específico de velocidad también puntuarán alto en el factor velocidad del cuestionario QAR - Precon.

\subsection{La elección de la muestra.}

La elección de la muestra está sujeta a las características de los profesionales que deben aplicar este tipo de programas en las autoescuelas: los profesores de formación vial. Para poder aplicar programas de este tipo, se debe formar a los profesionales en aquellas competencias necesarias para trabajar el cambio de actitudes. De este modo, se realizaron cursos de de formación de formadores a profesores de formación vial para enseñar a aplicar el programa de autocontrol de la velocidad, así como otros programas relacionados con otros factores de riesgo, a preconductores en fase de aprendizaje para la obtención del permiso de conducir.

Los criterios de selección para la impartición de cursos se realizaron en función del factor geográfico y proporcional al $\mathrm{n}^{\circ}$ de autoescuelas existentes en Cataluña. En total se realizaron 10 cursos con un promedio de 15 profesores de formación vial en cada uno de ellos (un total de 150 profesores), repartidos entre las provincias de Lleida, Girona, Tarragona y Barcelona.

De las casi 140 autoescuelas (poco más del $10 \%$ de las existentes en Cataluña), cuyos profesores participaron en los cursos de formación, seleccionadas según el estrato geográfico y de representatividad, solo 46 apli- caron el programa de mejora del autocontrol de velocidad objeto de esta investigación.

La selección del programa a aplicar no se realizó al azar entre las 140 autoescuelas, sino que su elección responde a los resultados obtenidos según el perfil de riesgo observado en la aplicación del QAR-Precon, puesto que el objetivo de la intervención en preconductores era el de reducir el nivel de riesgo en aquellos factores que presentaban algún tipo de problemática específica.

Dado que no han podido formar parte de la muestra todos los profesores de formación vial, debido a su falta de calificación para aplicar cursos de seguridad vial inspirados en un modelo de cambio de actitudes y que los preconductores que han participado en el curso no han podido ser elegidos al azar, por los motivos anteriormente explicados, hay que ser prudentes y entender que los datos serán solo generalizables en el caso de que la población integre las variables básicas de la muestra utilizada.

Para la selección del grupo control se pidió la colaboración a 15 profesores de autoescuela para que aplicaran dentro de la misma quincena el pretest y postest a los alumnos en las clases teóricas en el mismo momento de la aplicación del programa.

\subsection{Aspectos descriptivos de la mues- tra.}

Los sujetos proceden de 46 autoescuelas, el $53,9 \%$ son hombres y el $46,1 \%$ mujeres. Las edades se reparten de la siguiente manera: 0,4\% tienen menos de 18 años, el 74,7\% se encuentra en el intervalo de 18 - 24 años, el $18,7 \%$ se sitúa entre los 25 - 35 años, el 6,2\% tiene más de 35 años.

El 48,9\% de los sujetos conduce habitualmente bicicletas. La media de kilómetros que realizan en bicicleta es de 5,55. La media de accidentes/año de los jóvenes utilizando este vehículo es de aproximadamente 2. 
El 29,5\% de los sujetos conduce habitualmente ciclomotores. La media de kilómetros que realizan en ciclomotor es de 71,77. La media de accidentes/año de los sujetos que conducen ciclomotor es de 1,3.

El 4,7\% de los sujetos ha manifestado que conduce otro tipo de vehículos. La media de quilómetros que realizan en otro tipo de vehículos es de 67,33. La media de accidentes/año utilizando otro tipo de vehículos es de aproximadamente 1.

Finalmente la media de accidentes/año como peatón de los encuestados es de 0,7.

\subsection{Extracción de factores.}

El primer paso en la investigación ha sido comprobar si es aplicable el análisis facto- rial, para ello se ha calculado la medida de adecuación muestral de Kaiser - Meyer Olkin (KMO) y se ha aplicado el test de esfericidad de Barlett (.885) utilizado para probar si la matriz de correlaciones es la identidad. El índice KMO arroja un valor de 0,885 (se considera en este caso aceptable puesto que pertenece al intervalo $0.7-0.8$ ). El test de esfericidad de Bartlett nos permite rechazar la hipótesis nula (interdependencia entre las variables), lo que ratifica el resultado obtenido con la medida KMO. Todo ello nos lleva a concluir que el análisis factorial que sigue a continuación resulta pertinente y puede proporcionarnos conclusiones satisfactorias (ver tabla 3).

Tabla 3: Prueba KMO y prueba de Bartlett.

\begin{tabular}{|lc|c|}
\hline \multicolumn{2}{|l|}{ Medida de adecuación muestral de Kaiser-Meyer-Olkin. } &, 885 \\
\hline Prueba de esfericidad de Bartlett & Chi-cuadrado aproximado & 696,796 \\
& gl & 66 \\
& Sig. &, 000 \\
\hline
\end{tabular}

También se facilitan las comunalidades, que nos proporcionan una medida del grado de explicación de cada variable a través de los 12 factores. Todas las comunalidades son iguales a 1 lo que pone de manifiesto que el grado de explicación es satisfactorio (véase tabla 4).

Tabla 4: Comunalidades

\begin{tabular}{|l|c|c|}
\hline & Inicial & Extracción \\
\hline 1. Me gusta correr & 1,000 &, 399 \\
2. Voy a $+15 \mathrm{~km} / \mathrm{h}$ de la velocidad adecuada & 1,000 &, 524 \\
3. Iré a $+50 \mathrm{~km} / \mathrm{h}$. por ciudad & 1,000 &, 407 \\
4. Tendré habilidad para correr con facilidad & 1,000 &, 465 \\
5. Correré más por vías conocidas & 1,000 &, 466 \\
6. Tendré facilidad para correr en autopista & 1,000 &, 343 \\
7. La velocidad me parece atractiva & 1,000 &, 543 \\
8. Utilidad de los turismos actuales para correr & 1,000 &, 442 \\
9. Correr para tener mayor calidad de vida & 1,000 &, 485 \\
10. Me gusta que quien conduzca corra & 1,000 &, 284 \\
11. Evaluación de un conductor por la velocidad & 1,000 &, 494 \\
12. Si me esfuerzo podré correr más & 1,000 &, 590 \\
\hline
\end{tabular}


En la tabla 8 se muestran todos los factores que se obtienen con el método de componentes principales (ACP) y se ha procedido a una rotación ortogonal para ganar en interpretabilidad (los autovalores y el porcentaje de varianza explicado por cada factor después de la rotación también figuran en la tabla 8). En el ACP se seleccionan aquellos factores que tienen un valor propio asociado mayor a 1. En este caso se han encontrado tres factores tal y como puede comprobarse en la tabla 5. Estos tres factores explican el $64,889 \%$ de la varianza de todos los ítems.
Con este modelo de tres factores las comunalidades se reparten de forma homogénea entre los ítems (de 0,284 a 0,590). El primer factor explica un $45,3 \%$ de la varianza de la muestra, el segundo factor explica el $11,192 \%$ y el tercer factor el $8,352 \%$, estos tres factores son capaces de explicar conjuntamente casi un $65 \%$, lo que se puede interpretar como un porcentaje aceptable. Dicho de otro modo, con menos un 35\% de pérdida de información, se pueden expresar cada una de las variables como una combinación lineal de estos tres factores (consultar tabla 5).

Tabla 5: Varianza total explicada por los tres factores

\begin{tabular}{|c|c|c|c|c|c|c|c|c|c|}
\hline \multirow[b]{2}{*}{ Componente } & \multicolumn{3}{|c|}{ Autovalores iniciales } & \multicolumn{3}{|c|}{$\begin{array}{l}\text { Sumas de las saturaciones al } \\
\text { cuadrado de la extracción }\end{array}$} & \multicolumn{3}{|c|}{$\begin{array}{l}\text { Suma de las saturaciones al } \\
\text { cuadrado de la rotación }\end{array}$} \\
\hline & Total & $\begin{array}{c}\% \text { de la } \\
\text { varianza }\end{array}$ & $\begin{array}{c}\% \\
\text { acumulado }\end{array}$ & Total & $\begin{array}{c}\% \text { de la } \\
\text { varianza }\end{array}$ & $\begin{array}{c}\% \\
\text { acumulado }\end{array}$ & Total & $\begin{array}{c}\% \text { de la } \\
\text { varianza }\end{array}$ & $\begin{array}{c}\% \\
\text { acumulado }\end{array}$ \\
\hline 1 & 5,441 & 45,345 & 45,345 & 5,441 & 45,345 & 45,345 & 3,015 & 25,128 & 25,128 \\
\hline 2 & 1,343 & 11,192 & 56,537 & 1,343 & 11,192 & 56,537 & 2,810 & 23,413 & 48,541 \\
\hline 3 & 1,002 & 8,352 & 64,889 & 1,002 & 8,352 & 64,889 & 1,962 & 16,348 & 64,889 \\
\hline 4 & ,744 & 6,202 & 71,091 & & & & & & \\
\hline 5 & 635 & 5,289 & 76,380 & & & & & & \\
\hline 6 & ,571 & 4,759 & 81,139 & & & & & & \\
\hline 7 & ,522 & 4,347 & 85,486 & & & & & & \\
\hline 8 & ,424 & 3,530 & 89,016 & & & & & & \\
\hline 9 & ,405 & 3,375 & 92,391 & & & & & & \\
\hline 10 & 338 & 2,817 & 95,208 & & & & & & \\
\hline 11 & ,301 & 2,506 & 97,714 & & & & & & \\
\hline 12 & ,274 & 2,286 & 100,000 & & & & & & \\
\hline
\end{tabular}

Método de extracción: Análisis de Componentes principales.

Tras la rotación se obtiene una estructura simple bastante clara con las siguientes excepciones (tal y como puede comprobarse en la tabla 6):

$\varnothing$ Los ítems "Pienso que los turismos actuales son para correr, por eso tienen las prestaciones que tienen" y "Creo que en autopista y otras vías rápidas me será fácil correr" presentan saturaciones elevadas en los factores 1 (.552 y .428) y 3 (.591 y .583), en ambos casos más elevados que en el factor 2, lo que indica que estos dos aspectos están relacionados con lo que miden el factor 1 y el factor 2 .

$\varnothing$ El ítem “Me gusta correr” pesa en los factores 2 (.668) y 3 (.449), lo que indica que este aspecto esta relacionado con lo que miden el factor 2 y el factor 3.

Se puede aceptar la existencia de tres factores que justifican la interdependencia de las variables tal y como muestra la matriz de componentes rotados de la tabla 6 . 
Tabla 6: Matriz de componentes rotados

\begin{tabular}{|l|c|c|c|}
\hline & \multicolumn{3}{|c|}{ Componente } \\
\cline { 2 - 4 } & 1 & 2 & 3 \\
\hline Evaluación de un conductor por la velocidad &, 797 &, 270 &, 026 \\
Correr para tener mayor calidad de vida &, 754 &, 142 &, 246 \\
Si me esfuerzo podré correr más &, 682 &, 250 &, 370 \\
Voy a +15 km/h de la velocidad adecuada &, 674 &, 287 &, 232 \\
Tendré facilidad para correr en autopista &, 192 &, 779 &,- 020 \\
La velocidad me parece atractiva &, 283 &, 753 &, 217 \\
Me gusta correr &, 046 &, 668 &, 449 \\
Tendré habilidad para correr con facilidad &, 488 &, 666 &,- 093 \\
Iré a + 50 km/h. por ciudad &, 175 &, 659 &, 284 \\
Me gusta que quien conduzca corra &, 126 &, 136 &, 825 \\
Utilidad de los turismos actuales para correr &, 552 &, 058 &, 591 \\
Correré más por vías conocidas &, 428 &, 230 &, 583 \\
\hline
\end{tabular}

Método de extracción: Análisis de componentes principales.

Método de rotación: Normalización Varimax con Kaiser.

a. La rotación ha convergido en 8 interacciones

A continuación se describen las características más significativas de cada uno de los factores encontrados.

Factor 1, lo denominamos velocidad como valor personal, puesto que las variables que están más correlacionadas con este factor son:

$\varnothing$ Pienso que un buen conductor o conductora debe saber correr.

Ø Si aprendo a correr me ayudará a vivir mejor

Ø Si me esfuerzo en conducir bien podré correr más

Ø Creo que iré a $15 \mathrm{~km} / \mathrm{h}$ más de la velocidad señalada.

$\varnothing$ Pienso que los turismos actuales son para correr, por eso tienen las prestaciones que tienen.

Todos los ítems de este factor presentan una correlación positiva, lo que pone de manifiesto que un valor elevado de este factor implica un alto nivel de riesgo en los aspectos evaluados. Este factor se hace eco del aprendizaje de la velocidad considerado como un valor positivo y una meta a alcanzar en la vida de los jóvenes.

Factor 2, lo denominamos velocidad como forma de vida, puesto que las variables que están más correlacionadas con este factor son:
Ø Creo que en autopista y otras vías rápidas me será fácil correr.

$\varnothing$ Para mi uno de los mejores atractivos de la conducción es la velocidad.

$\varnothing$ Me gusta correr

$\varnothing$ Creo que tengo habilidades para correr con facilidad

$\varnothing$ Si puedo, por ciudad iré a más de 50 $\mathrm{km} / \mathrm{h}$.

Todos los ítems de este factor presentan una correlación positiva, lo que pone de manifiesto que un valor elevado de este factor implica un alto nivel de riesgo en los aspectos evaluados. Este factor se hace eco de la velocidad como parte importante de la vida de los jóvenes.

Factor 3, lo denominamos falsa percepción de seguridad basada en elementos externos, puesto que las variables que están más correlacionadas con este factor son:

$\varnothing$ Cuando voy de acompañante me gusta que el conductor corra.

$\varnothing$ Pienso que los turismos actuales son para correr, por eso tienen las prestaciones que tienen.

$\varnothing$ Pienso que correré más en las vías conocidas.

$\varnothing$ Me gusta correr.

Todos los ítems de este factor presentan una correlación positiva, lo que pone de manifiesto que un valor elevado de este factor 
implica un alto nivel de riesgo en los aspectos evaluados. Este factor se hace eco de los elementos que proporcionan una falsa seguridad en la conducción. Las prestaciones de los vehículos no pueden controlar el riesgo en situaciones de exceso de velocidad y el conocimiento de la vía no permite controlar las incidencias del tráfico.

En resumen, se ha conseguido sustituir los 12 ítems del cuestionario por tres factores, cada uno de los cuales incide en los tres aspectos más claramente diferenciables de la velocidad (velocidad como valor personal, velocidad como forma de vida y falsa percepción de seguridad en relación con velocidad basada en elementos externos).

\subsection{Efecto del programa de cambio de} actitudes en relación con la velocidad. Aplicación de un modelo de medidas repetidas.

El primer paso ha sido comprobar si las matrices de las varianzas - covarianzas de los niveles del factor intra - sujetos son iguales en cada uno de los niveles del factor inter - sujetos. El estadístico M. de Box permite contrastar la hipótesis de igualdad entre matrices de varianzas - covarianzas. Se observa en la tabla 7, dado que el la significación es 0.000 , resulta que la estructura de covarianza para los distintos grupos es diferente, es decir, no todos los grupos tienen la misma matriz de covarianzas.
Tabla 7: Prueba de Box sobre la igualdad de las matrices de covarianzas.

\begin{tabular}{|l|r|}
\hline M de Box & 32,904 \\
F & 10,825 \\
gl1 & 3 \\
gl2 & 271960,6 \\
Significación &, 000 \\
\hline
\end{tabular}

Contrasta la hipótesis nula de que las matrices de covarianza observadas de las variables dependientes son iguales en todos los grupos.

a. Diseño: Intercept+Grup

Diseño intra sujetos: velocidad

Dados los resultados de la prueba $\mathrm{M}$ de Box, se ha optado por utilizar la prueba de la F conservadora tal y como recomienda Riba (1990).

Al realizar la correlación entre el nivel de riesgo obtenido en el pretest y el postest todos los ítems presentan una correlación positiva en grupo control ( $\mathrm{p}=0,000$ y $\mathrm{r}$ de Pearson $=0,793)$ y en el grupo experimental $(\mathrm{p}=$ 0,000 y r de Pearson $=0,451$ ), lo que pone de manifiesto que un valor elevado de este factor implica un alto nivel de riesgo en los aspectos evaluados.

Se ha realizado un análisis de factorial intra - sujetos para comprobar la evolución de la variable velocidad en los dos grupos (control y experimental). Si observamos los resultados presentados en la tabla $\mathrm{x}$, comprobamos que la evolución de la variable velocidad es distinta según el grupo al que pertenecen los sujetos que han participado en esta investigación, tal como nos muestra el nivel de significación de la F conservadora (limite inferior) que es de 0.000 (ver tabla 8). 
Tabla 8: Prueba de efectos intra - sujetos para comprobar la evolución de la variable velocidad en los dos grupos.

\begin{tabular}{|c|c|c|c|c|c|c|}
\hline Fuente & $\begin{array}{l}\text { Suma de cuadrados } \\
\text { tipo III }\end{array}$ & gl & $\begin{array}{c}\text { Media } \\
\text { cuadrática }\end{array}$ & $\mathrm{F}$ & $\begin{array}{l}\text { Signifi- } \\
\text { cación }\end{array}$ & $\begin{array}{l}\text { Po- } \\
\text { tencia } \\
\text { ob- } \\
\text { serva- } \\
\text { da }^{\mathrm{a}} \\
\end{array}$ \\
\hline Velocidad Límite-inferior & 1747,701 & 1,000 & 1747,701 & 117,957 &, 000 & 1,000 \\
\hline Velocidad * Grup Límite-inferior & 1089,418 & 1,000 & 1089,418 & 73,528 & 000 & 1,000 \\
\hline Error(velocidad) Límite-inferior & 3200,347 & 216,000 & 14,816 & & & \\
\hline
\end{tabular}

${ }^{\mathrm{a}}$ Calculado con alfa $=, 05$

La interacción velocidad - grupo es significativa, pero los resultados obtenidos en las pruebas anteriores sugieren que el valor $\mathrm{p}$ no es concluyente. Por ello se decide aplicar dos pruebas t para medidas repetidas con la corrección de Bonferroni para comparaciones múltiples para constatar cual ha sido el efecto del programa de cambio de actitudes en los preconductores, de esta manera se evita cometer errores de Tipo 1.
En la tabla 9 obtenemos los resultados de las comparaciones por pares entre los niveles del factor programa de cambio de actitudes en relación con el factor de riesgo velocidad. El resultado de estas comparaciones indica que el grupo experimental en el nivel 2 (postest) es significativamente mejor (Sig. = 0.000 ) que en el nivel 1 (pretest) y que el grupo experimental no se producen mejoras $($ Sig. $=0.177)$.

Tabla 9: Resultados de las comparaciones por pares para comprobar los efectos del programa de cambio de actitudes Medida: MEASURE_1

\begin{tabular}{|c|c|c|c|c|c|c|c|}
\hline \multirow[b]{3}{*}{ Grupo } & \multirow{3}{*}{\multicolumn{2}{|c|}{ (J) velocidad }} & \multicolumn{5}{|l|}{ Estadísticos } \\
\hline & & & \multirow{2}{*}{$\begin{array}{c}\text { Diferencia } \\
\text { entre medias } \\
\text { (I-J) }\end{array}$} & \multirow[b]{2}{*}{ Error típ. } & \multirow[b]{2}{*}{ Significación $^{\mathrm{a}}$} & \multicolumn{2}{|c|}{$\begin{array}{c}\text { Intervalo de confianza } \\
\text { al 95\% pata la diferen- } \\
\text { cia }^{\text {a }}\end{array}$} \\
\hline & & & & & & $\begin{array}{l}\text { Límite } \\
\text { inferior }\end{array}$ & $\begin{array}{l}\text { Límite } \\
\text { superior }\end{array}$ \\
\hline Grupo Control & 1 & 2 & 930 & 686 & 177 &,- 422 & 2,821 \\
\hline Grupo Experimental & 1 & 2 & $7,904 *$ & ,437 & ,000 & 7,042 & 8,766 \\
\hline
\end{tabular}

Basadas en las medias marginales estimadas.

* La diferencia de las medias es significativa al nivel ,05

a Ajuste para comparaciones múltiples: Bonferroni.

Por último el modelo incluye un gráfico del perfil, donde se observa claramente que el nivel de riesgo en el grupo experimental disminuye después de la realización del pro- grama de cambio de actitudes. Se observa también con claridad el nivel de riesgo casi no disminuye en situación postest en el grupo control. 
Grafico 1: Grafico de perfil representado el efecto del programa de cambio de actitudes en relación con la velocidad.

\section{Medias marginales estimadas de MEASURE_1}

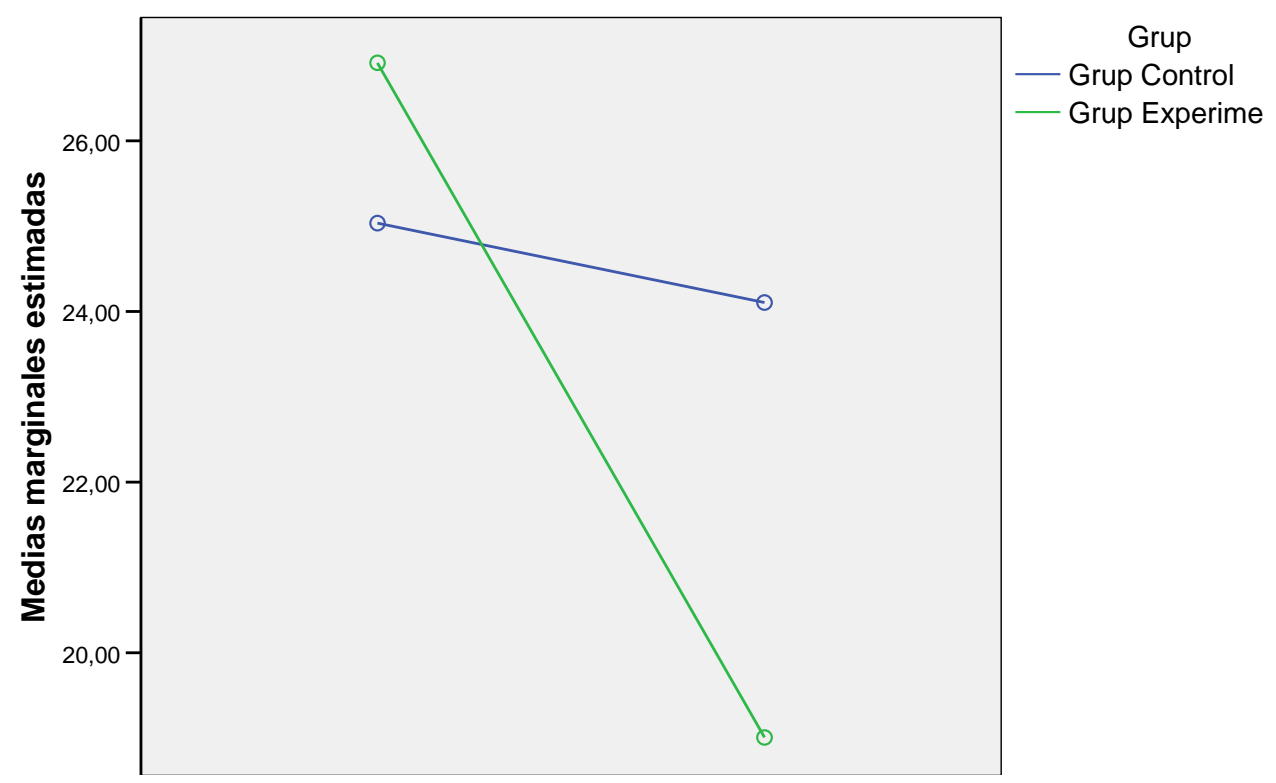

Con todo el análisis realizado con la aplicación del modelo de medidas repetidas para conocer el efecto del programa de cambio de actitudes en relación con la velocidad, se concluye que:

1. Existen diferencias significativas en el grupo experimental entre la evaluación realizada en el pretest y la desarrollada en el postest, con una diferencia de medias de 7,9 puntos que, con un $95 \%$ de probabilidad es de entre 7 y 8,77 puntos en la población, con lo que en el postest se ha disminuido claramente la puntuación obtenida en el postest.

2. No existen diferencias significativas en el grupo control entre la evaluación realizada en el pretest y la aplicada en el postest, por lo que la diferencia obtenida entre el pretest y el postest $(0,930)$ puede ser debida simplemente al azar del muestreo.

\section{DISCUSIÓN.}

Estos resultados se han obtenido a partir del trabajo y compromiso del $10 \%$ de las autoescuelas de Cataluña, con lo que los resultados deben leerse como tendencias si se tiene en cuenta el muestreo realizado. Pero, estos resultados no son fruto del azar, la larga trayectoria de nuestro equipo de investigación en aplicación de cursos de seguridad vial basados en el modelo de cambio de actitudes presentado en este artículo, que desde el año 1992 esta aplicando e investigando este modelo avalan el impacto y fuerza del modelo en el cambio de actitudes. Todos estos resultados han facilitado que el modelo de cambio de actitudes presentado en este artículo sea el que el Servei Català de Trànsit de la Generalitat de Cataluña, utilice este modelo en la aplicación de los cursos para la recuperación de puntos del permiso de conducir que nuestro equipo de investigación supervisa.

El programa de cambio de actitudes aplicado a jóvenes preconductores que mostraban un perfil de riesgo alto en velocidad consigue disminuir el riesgo de sufrir un accidente debido a la velocidad, presentando un nivel de riesgo moderado una vez concluido el programa. Por tanto es posible cambiar 
actitudes en los jóvenes en relación al factor de riesgo velocidad. Aunque seria importante demostrar el impacto de este cambio al cabo de un cierto tiempo.

Los jóvenes que realizaron una formación de tipo tradicional no consiguieron mejorar su nivel de seguridad en relación con la velocidad; es más estos conductores mostraban un nivel de riesgo alto en velocidad y se ha mantenido una vez finalizado el proceso formativo. Con lo que la formación tradicional que se desarrolla en las autoescuelas puede no incidir en el cambio de actitudes.

La investigación pone de manifiesto que el diseño, estructura y desarrollo del Programa de Conducción Segura para Preconductores (PCS - Precon) basado en el modelo de cambio de actitudes propuesto puede mejorar la seguridad en los jóvenes preconductores.

Las autoescuelas que se decidan por la implementación programas de seguridad vial para preconductores (basando la intervención en una evaluación inicial del perfil de riesgo, introduciendo información individualizada y adaptada al grupo a partir de los datos obtenidos con el cuestionario, revisando las destrezas y hábitos de seguridad y trabajando la mejora de emociones y valores de seguridad) pueden contribuir a la formación de conductores sensibilizados por una conducción segura $\mathrm{y}$, por tanto, a la disminución de los accidentes y sus consecuencias, y demostrar que es posible cambiar actitudes en los jóvenes.

Para poder cambiar actitudes en los jóvenes en relación con la velocidad se debe tener en cuenta que la velocidad se compone de tres elementos principales:

- Es un valor personal y una meta a alcanzar por muchos jóvenes. Este aspecto hace que, en muchas ocasiones, deban tocarse aspectos emotivos o de valores en el momento de intentar cambiar actitudes utilizando procesos formativos para reducir los accidentes de tráfico.
- Para muchos jóvenes la velocidad es una forma de vida y por ello un hábito adquirido, con lo que es necesario la revisión y reflexión sobre los comportamientos de los jóvenes.

- Algunos jóvenes manifiestan una falsa percepción de la seguridad frente a la velocidad (elementos de seguridad de los vehículos y las actuales vías), lo que denota la importancia de la información en el momento de poder incidir en el cambio de actitudes de los jóvenes.

Estos tres elementos o factores se encuentran detallados en las fases del modelo de cambio de actitudes propuesto, a saber: el sesgo o la incorrección de la información (relacionado con la falsa percepción de seguridad), los valores o emociones que despierta la velocidad en los jóvenes y los comportamientos o hábitos de estos. El análisis factorial ha corroborado la importancia de estos tres elementos.

De esto se deduce que el proceso formativo para el cambio de actitudes debe contener la información necesaria para que los jóvenes adquieran conciencia de la importancia del factor de riesgo, su incidencia en la accidentalidad y en la gravedad de los accidentes de tráfico, así como las medidas preventivas que ayudaran a reducir este riesgo. La información por si sola, no ayudará a cambiar actitudes, será necesario incluir en el proceso formativo la revisión de comportamientos y la adquisición de hábitos de seguridad en relación con el factor de riesgo, así como elementos relacionados con los valores personales y las emociones que ayuden a dar sentido a la información y los hábitos.

A nuestro entender, la diferencia entre las autoescuelas que sólo realicen una formación basada en el modelo tradicional y las que incorporen programas de cambio de actitudes para preconductores debería constatarse a partir del seguimiento de sus alumnos en relación con el número de accidentes y la intensidad de los daños que éstos puedan tener como conductores. La experiencia que 
nuestro grupo de investigación tiene al respecto muestra resultados esperanzadores en relación con la mejora de la seguridad vial a través de programas formativos que se fundamenten en este modelo de cambio de actitudes propuesto y analizado en este artículo.

Por ultimo, queremos ratificar una de las limitaciones de esta investigación. No han podido formar parte de la muestra todos los profesores de formación vial, debido a su falta de calificación para aplicar cursos de seguridad vial inspirados en un modelo de cambio de actitudes, además los preconductores que han participado en el curso tampoco han podido ser elegidos al azar. Por ello debemos ser prudentes y entender que estos resultados solo podrán ser generalizables en el caso de que la población integre las variables básicas de la muestra utilizada. Sin embargo, los resultados obtenidos nos ofrecen un futuro esperanzador para la disminución de la mortalidad en las carreteras catalanas.

\section{REFERENCIAS BIBLIOGRÁFI- CAS}

Abelson, R.P. y Rosenberg, M.J (1958). Symbolic physiologic: a model of attitudinal cognition. Behaviour Science, 3, 1-13.

Abelson, R.P. (1983). Whatever became of consistency theory?. Personality and Social Psychology Bulletin. 9, 37-54.

Ajzen, I. \& Fishbein, M. (Eds.) (1980). Understanding attitudes and predicting social behaviour. Englewood Cliffs, NJ: PrenticeHill.

Ajzen, I, \& Fishbein, M. (2000). Attitudes and the attitude-behavior relation: reasoned and automatic processes. En W. Stroebe, M Hewstone. (Eds.) European Review of Social Psychology (Vol. 11, 1-33). Chichester: Wiley

Ajzen, I. and Fisbein M. (2005). The influence of Attitudes on Behavior in Dolores Albarración, B, T. Johnson and M.P. Zanna (Eds) The Handbook of Attitudes. London: Lawrence Erlbaum Associates.
Dolores Albarración, B, T. Johnson and M.P. Zanna (Eds). London: Lawrence Erlbaum Associates.

Albarracín, D., Johnson, B.T. \& Kumkale, G.T. (Eds.) (2005). The Handbook of Attitudes. Mahwah, NJ: Erlbaum

Alhakami, A.S. \& Slovic, P. (2004). A psychological study of the inverse relationship between perceived risk and perceived benefit. Risk Analysis. 14, 1085-1096.

Allport G.W. (1954). The nature of prejudice. Garden City, NY: Doubleday Anchor Books

Aronson E. (1992). The return of the repressed: Dissonance theory makes comeback. Psychological Inquiry, 3, 303-311

Beauvois, J.L. \& Joule, R.V. (1996). A radical dissonance theory. Bristol, PA: Taylor \& Francis.

Bisquerra Alzina, R. (2003). Educación emocional y competencias básicas para la vida. Revista de Investigación Educativa. 21, nº $1,7-46$.

Cooper, J. (1977). The arousal of dissonance and responsibility for aversive events. C. A. Kiesler (Chair) Twenty years later: current perspectives on cognitive dissonance Symposium presented at the meeting of the Society of Experimental Social. Psychology. Austin Texas.

Erwin, P. (2001). Attitudes and persuasión. New York: Psychology press

Evans, L. (1985). Human behaviour feedback and traffic safety. Human Factors. 27, 555-576.

Feldman, S. (Ed.) (1966). Cognitive consistency. New York: Academic Press.

Festinger, L. (1957). A Theory of cognitive dissonance. Stanford, CA: Stanford University Press

Festinger, L. \& Carlsmith, J.M. (1959). Cognitive consequences of forced compliance. Journal Abnormal Soc. Psychology. 58, 201-210.

Fishbein, M. (1963). An investigation of the relationship between beliefs about and object and the attitude toward that object. $\mathrm{Hu}$ man Relations. 16, 233-240. 
Heider, F. (1958). The psychology of interpersonal relations. New York: Wiley.

Hoyt, K.B. (1972). Introduction. En J.E. Bottoms (Ed.) Career Education Resource Guide. New Jersey: General Learning Coroporation. 1-10.

Jariot, M. y Rodríguez, D. (2005). Evaluación y mejora de las competencias del formador vial desde un modelo de cambio de actitudes. Actas del XII Congreso Nacional de Modelos de Investigación Educativa. Investigación en Innovación Educativa. Asociación Interuniversitaria De Investigación Pedagógica (AIDIPE). La Laguna. España.

Jariot, M. y Rodríguez, M. (2006). Les compències del profesor de formació viària per a l'educació de joves conductors des d'un model de canvi d'actituds. El paper de les autoescoles.. Actas del IV Congrés Internacional sobre polítiques europees de trànsit. La formació, eina de prevenció viària. Barcelona. Servei Català de Trànsit. Generalitat de Catalunya. Departament d'Interior

Kelman, H.C. (1953). Attitude change as a function of response restriction. Human Relations. 6, 185-214.

McDaniels, T., Kamlet, S.K., y Fischer, G.W. (1992). Risk Perception and the Valour of Safety. Risk Analysis. 12, n²4, 495503.

McGuire, W.J. (1964). Inducing resistance to persuasion: Some contemporary approaches. En L. Berkowitz (Ed.), Advances in experimental social psychology (Vol. 1, pp. 191-229) New York: Academic Press

Montané, J., Ferrer, F. (1993). Cuaderno de formación de formadores del conductor. Programa de educación del conductor. P.E.C. Barcelona: PPU.

Montané, J. y Jariot, M. (2003). La psicología aplicada a una conducció segura i eficaç. Barcelona: Generalitat de Catalunya: Departament d'Interior. Servei Català de trànsit. (Grup ERESv UAB)

Montané, J., Jariot, M., Martínez, M., Amador, M. y Rodríguez M. (2003). Seguretat viària, conducció econòmica, medi ambient i contaminació. (2-150) Barcelona: Barce- lona: Generalitat de Catalunya: Departament d'Interior. Servei Català de trànsit. (Grup ERESv UAB)

Montané, J., (2004). Les dues maneres d'entendre el model de canvi de actitud, automatismes, costums i adquisició d'hàbits. La psicologia aplicada a una conducció segura y eficaç. Curs per a l'obtenció del certificat d'aptitud de profesor de formació viària. Convocatoria 2006. Barcelona: Generalitat de Catalunya: Departament d'Interior. Servei Català de trànsit. (Grup ERESv UAB)

Montané, J., Jariot, M. y Martínez, M. (2004). Models, instruments i processos d'intervenció per a la reducció de l'accidentalitat. "La formació dels formadors viaris" Actas del III Congrés Internacional sobre polítiques europees de trànsit. L'educació i la formació per a la mobilitat segura. Barcelona.Servei Català de Trànsit. Generalitat de Catalunya. Departament d'Interior

Montané, J., Jariot, M. (2005). Visió integrada de l'accident. El factor humà present en els agents de risc i seguretat. La psicologia aplicada a una conducció segura i eficaç. (p.110) Barcelona: Generalitat de Catalunya: Departament d'Interior. Servei Català de trànsit. (Grup ERESv UAB).

Montané, J., Jariot, M. y Rodríguez, M. (2007). Actitudes, cambio de actitudes y conducción segura. Un enfoque crítico aplicado a la reducción de los accidentes de tráfico. Barcelona: Laertes

Montané, J., Jariot, M. y Rodríguez, M. (2007). Qüestionari d'Avaluació de les competències del formador viari (Acomform). Barcelona: Barcelona: Generalitat de Catalunya: Departament d'Interior. Servei Català de trànsit. (Grup ERESv UAB)

Näätänen, R. \& Summala, H. (1974). A Model for the Role of Motivational Factors in Drivers Decision-Making. Accident Analysis and Prevention. 6, 243-261

Näätänen, R. \& Summala, H. (1976). Road user behaviour and traffic accidents. Amsterdam: North Holland Publishing Company. 
OECD Road Transport Research (1994). Improving road safety by attitude modification. París: OECD

Olson, J.M. \& Stone, J. (2005). The Influence of Behavior on Attitudes. En Dolores Albarracín, B.T., Johnson, y G.T. Kumkale (Eds.) The Handbook of Attitudes. (pp. 223272). Mahwah, NJ: Erlbaum

Osgood, C.E. \& Tannenbaum, P.H. (1955). The principle of congruity in the prediction of attitude change. Psychological Review. 62, 42-55.

Parker, D., Manstead, A. S.R., \& Stradling, S.G. (1995). Extending the theory of planned behaviour: The role of personal norm. British Journal of Social Psychology. 34, 127-137.

Peltzman, S. (1975). The effects of automobile safety regulation. Journal of Political Economy. 83, 677-725.

Peters, E.M., Burraston, B. \& Mertz, C.K. (2004). An Emotion-Based Model of Risk Perception and Stigma Susceptibility: Cognitive Appraisals of Emotion, Affective Reactivity, Worldviews, and Risk Perceptions in the Generation of Technological Stigma. Risk Analysis. 24, n5. 1349-1367.

Reason, J. (1990). Human Error. Cambridge: Cambridge University Press.

Riba Lloret, M.D. (1990). Modelo lineal de análisis de la varianza. Barcelona: Herder.

Rodríguez Parrón, M. (2001). Aportaciones de los predictores de riesgo a la educación vial en la escuela. Barcelona.: Servei de Publicacions de la Universitat Autònoma de Barcelona.

Stone, J. \& Cooper, J. (2001). A selfstandards model of cognitive dissonance. Journal of Experimental Social Psychology. 37, 228-243.

Thurstone (1931). The indifference function. Journal of Social Psychology. N.2. pp. 139167

Ulleberg P. (2002). Influencing subgroups of young drivers and their passengers. Motivational influences of personality traits on risk-taking attitudes and driving behaviour. [Doctoral dissertation, Norwegian University of Science and Technology] Oslo: Institute of Transport Economics.

Wicker, A.W. (1969). Attitudes versus actions: The relationship of verbal and overt behavioural responses to attitude objects. Journal of Social Issues. 25, 41-78.

Wicklund, R.A. \& Brehm, J.W. (1976). Perspectives on cognitive dissonance. Hillsdale, NJ: Lawrence Erlbaum Associates.

Wilde, G. J.S. (1998). Risk Homeostasis theory: an overview. Injury Prevention. 4, 89-91.

Wilde, G. J.S. (1994). Target Risk - Dealing with the danger of death, disease and damage in everyday decisions. Toronto: PDE Publications.

\section{ANEXO I}

\section{CUESTIONARIO DE EVALUACIÓN DEL RIESGO DEL PRECONDUCTOR (QAR-PREC)}

En el cuestionario encontrarás afirmaciones similares a estas:

\section{Afirmación A: Creo que la conducción tranquila es aburrida}

\section{((Si crees que conducir tranquilamente, sin correr es bastante aburrido, señala con una cruz la casilla del número 3 en la hoja de respuestas))}

Afirmación B: En caso de accidente evitaría el choque frontal aunque con la maniobra pudiera volcar el turismo.

Siempre $1-2 \quad 2 \quad-3 \quad-4 \quad$ Nunca

((Si crees que casi siempre podrías evitar un choque frontal, señala con una cruz la casilla del número 2 en la hoja de respuestas)). 
1. Hago ejercicio para estar en forma física.

$$
\text { I.- ¿Cuido de mi salud? }
$$

2. Tomo bebidas alcohólicas.

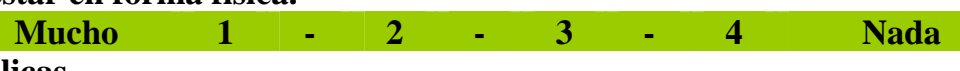

3. Fumo en lugares prohibidos.

Nunca $1 \quad-\quad 2 \quad-\quad 3 \quad-4 \quad 4 \quad$ Siempre

Nunca $1-2-3-3-4$ Siempre

4. En la conducción pienso tener cuidado de mi salud.

5. Como equilibradamente.

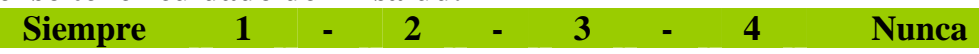

6. Duermo al menos 8 horas al día.

Siempre $1-2 \quad-3 \quad-4 \quad$ Nunca

II.- Los grandes factores de riesgo y seguridad

A) Alcohol.

7. Tomo bebidas alcohólicas sin pasar los límites legales.

Nunca $1-2-3-4$ Siempre

8. Mis compañeros toman bebidas alcohólicas.

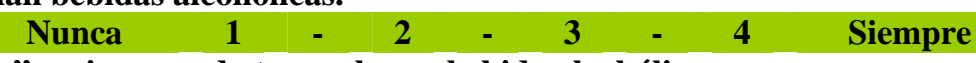

9. Creo que me “enrollo” mejor cuando tomo alguna bebida alcohólica.
Nunca $1-2 \quad-3-4 \quad$ Siempre

10. Tal como soy, creo que conduciré después de haberme tomado más de una copa, un cubata, dos cervezas, dos vasos de vino...

$\begin{array}{llllllll}\text { Nunca } & 1 & - & 2 & - & 3 & - & 4\end{array}$

11. Cuando haya bebido dos copas o más buscaré un/a conductor/a que no haya bebido.
Siempre
- 4 Nunca

$1 \quad-$

$2 \quad-\quad 3$

12. Tengo claro que si bebo no conduciré.

Totalmente de acuerdo $\quad 1-2-2 \quad-\quad 3-4 \quad$ Totalmente en desacuerdo

13. Creo que puedo conducir bien con más de dos copas o tres vasos de vino.

$$
\text { Nunca } 1-2-2-3-4 \text { Siempre }
$$

14. Cuando salgo de fiesta con los/las amigos/as es normal que bebamos y conduzcamos.

Totalmente en desacuerdo $1-2-2-3-4 \quad$ Totalmente de acuerdo

15. Me gusta sentir la velocidad.

B) Velocidad.
Nada
$1 \quad-\quad 2 \quad-\quad 3$
$-4$
Mucho

16. Me gusta sentir como la velocidad domina el vehículo.

$\begin{array}{llllllll}\text { Nada } & 1 & - & 2 & - & 3 & - & 4\end{array}$ Mucho

17. Tengo amigos/as amantes de la velocidad.

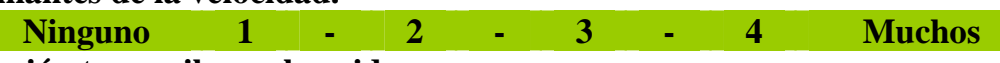

18. Creo que la conducción tranquila es aburrida.
Totalmente en desacuerdo
1
$2-3$

4 Totalmente de acuerdo

19. Creo que si el tráfico me lo permitiera correría más de la velocidad permitida.

$$
\begin{array}{lllllll}
\text { Nunca } & 1 & - & 2 & - & 3 & -
\end{array} \text { Siempre }
$$

20. Creo que correré más que los otros si el turismo me lo permite.
Nunca

1
C) Tendencia al riesgo.
Siempre

21. ¿Cuántas prácticas crees que deberías hacer para conducir con seguridad?
1 Más de 16
2 Entre 11 y 15
3 Entre 10 y 5

4
guridad?
tre 10 y 5

22. Ocasionalmente, me gusta arriesgarme para impresionar a los acompañantes.

23. Es excitante sentir el riesgo de vez en cuando.
Nada
$1-2$
$4 \quad$ Mucho

3 - 4 Mucho

24. Me gustaría hacer competiciones en zonas poco transitadas.
Nada
$1 \quad-\quad 2$
3

25. Me gustaría ser de los conductores que va pegado a los vehículos de delante.

26. Creo que conduciré al límite de la tolerancia.

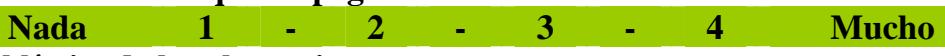

$\begin{array}{llll}\text { Nunca } & 1 & - & 2\end{array}$

$2-3-4 \quad$ Siempre


27. Creo que me clasificaré como un/a conductor/a atrevido/a.

\section{Totalmente en desacuerdo $1-2-2-3-4 \quad$ Totalmente de acuerdo}

\section{D) Seguridad proveniente prioritariamente del conductor}

28. Estoy mentalizado/a para evitar, sea como sea, el choque frontal aunque me salga de la vía.

$$
\begin{array}{lllllll}
\text { Mucho } & 1 & - & 2 & - & - & -
\end{array} \quad \text { Nada }
$$

29. En caso de accidente evitaría el choque frontal aunque con la maniobra pudiera volcar el turísmo.

$$
\text { Siempre } 1-2 \quad 2 \quad 3-4 \quad \text { Nunca }
$$

30. Me gustaría aprender a conducir de manera segura..

$$
\begin{array}{lllllll}
\text { Mucho } & 1 & - & 2 & - & - &
\end{array}
$$

31. Mi objetivo es sacarme el permiso de conducir más que aprender a conducir.

$\begin{array}{lllllll}\text { Totalmente en desacuerdo } & 1 & - & - & -4 & -4 & \text { Totalmente de acuerdo }\end{array}$

32. Fumaré mientras conduzca.

$$
\begin{array}{lllllllll}
\text { Nunca } & 1 & - & 2 & - & 3 & - & 4 & \text { Siempre }
\end{array}
$$

33. Quiero tener el permiso para poder salir de fiesta los fines de semana sin depender de nadie.

Totalmente en desacuerdo $1-2-3-4$ Totalmente de acuerdo

34. Me pone nervioso/a cuando los otros conductores van despacio.

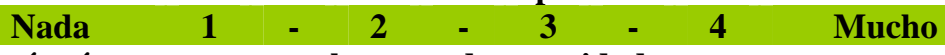

35. Con el cinturón correré más, ya que es un elemento de seguridad.

$\begin{array}{lllllllll}\text { Totalmente en desacuerdo } & 1 & - & - & - & - & \end{array} \quad$ Totalmente de acuerdo

36. Estoy de acuerdo con el permiso por puntos.

$\begin{array}{lllllllll}\text { Totalmente de acuerdo } & 1 & - & 2 & - & - & - & 4 & \text { Totalmente en desacuerdo }\end{array}$

37. Creo que podré conducir tres horas seguidas sin parar.

Nunca $\quad 1 \quad-2 \quad 2-3-4 \quad-4$ Siempre

38. Cuando tenga sueño, creo que pararé a dormir.

Siempre $1-2 \quad-\quad 3 \quad-4 \quad$ Nunca

39. Conducir de de noche es tan seguro como conducir de día.

Totalmente en desacuerdo $\quad 1-2-3-4 \quad$ Totalmente de acuerdo

40. Me gustan los/las conductores/as que ponen la radio o el CD del turismo a "tope".

$\begin{array}{lllllllll}\text { Totalmente en desacuerdo } & 1 & - & - & - & - & \end{array} \quad$ Totalmente de acuerdo

41. Creo que soy de los que hablarán por el móvil conduciendo.

$$
\text { Nunca } 1 \quad-2 \quad-3 \quad 3 \quad 4 \quad \text { Siempre }
$$

42. Tomo fármacos regularmente para reducir la ansiedad o para calmar el dolor.

$\begin{array}{lllllll}\text { Nunca } & 1 & - & 2 & - & 3 & -\end{array}$

\section{E) Acompañante}

43. Normalmente cuando vaya con el turismo llevaré a: (señala solo una)
1 Padres
2 Iré solo/a
3 Amigos/as
$4 \quad$ Amigos/as de noche

44. Pongo la música a todo volumen en el turismo cuando voy de acompañante.

$$
\begin{array}{lllllll}
\text { Nunca } & 1 & - & 2 & - & 3 & -
\end{array} \text { Siempre }
$$

45. Diré a los/las acompañantes que se pongan el cinturón.

Siempre $1-2 \quad 2-3 \quad-4 \quad$ Nunca

46. Creo que pasaré la revisión de la ITV.
F) Seguridad proveniente del vehículo.

$$
\text { Siempre } \quad 1 \quad-\quad 2 \quad-\quad 3 \quad-4 \quad \text { Nunca }
$$

47. Haré revisiones periódicas, una vez al año como mínimo, del estado de mi vehículo.
Siempre
1 -
$2-3-4$
Nunca

48. Según la proporción peso/potencia el vehículo que me quiero comprar o que conduciré es potencialmente:

$$
\text { Muy seguro } 1-2 \quad-\quad 3 \quad-4 \quad \text { Muy peligroso }
$$

49. El vehículo que conduciré tiene: (señala sólo una)
1 Menos de 2 años $2 \quad$ Entre 3 y 5 años
$3 \quad$ Entre 68 años
$4 \quad$ Más de 8 años

50. ¿Utilizaras otros vehículos?

$\begin{array}{llllllllll}\text { Infrecuentemente } & 1 & - & 2 & - & 3 & - & 4 & \text { Frecuentemente }\end{array}$

51. Me gustaría probar los límites de mi turismo.
Nunca
$1-2$
$2 \quad-3 \quad-\quad 4 \quad$ Siempre

52. Me gustaría tener un turismo que pesase poco y tuviera mucha potencia.

$\begin{array}{lllllll}\text { Nada } & 1 & - & 2 & - & 3 & -\end{array}$

53. Llevaré el móvil conectado cuando conduzca.

$\begin{array}{lllllllll}\text { Nunca } & 1 & - & 2 & - & 3 & - & 4 & \text { Siempre }\end{array}$


54. Si tuviera un turismo antiguo o con algunos problemas, iría a menos velocidad..

Siempre $1-2-3-4 \quad$ Nunca

55. Siempre me pongo el cinturón de seguridad de la misma forma.

56. Si voy en moto me pongo el casco.

Siempre $1-2-3-4 \quad$ Nunca

$\begin{array}{llllllll}\text { Siempre } & 1 & - & 2 & - & 3 & - & 4\end{array}$ Nunca

57. Si mi turismo tuviera sistemas de seguridad como el ABS o antiderrapante probaría a menudo como funcionan en situaciones límite.

$$
\text { Nunca } 1 \quad-\quad 2 \quad-\quad 3-4 \quad \text { Siempre }
$$

58. Si mi turismo fuera un GTI o similar haría muchos adelantamientos.

Nunca $1-2 \quad-3-4$ Siempre

G) Seguridad proveniente de la vía.

59. Reduciré la velocidad antes de entrar en un cruce o rotonda, aunque no haya indicaciones de reducción de velocidad.

Siempre $1-2 \quad-\quad 3 \quad-4 \quad$ Nunca

60. Por carretera, adelantaré a menos de 150 metros de una intersección.

$\begin{array}{lllllll}\text { Nunca } & 1 & - & 2 & - & 3 & -\end{array}$ Siempre

61. Entraré en las curvas con más velocidad de la señalizada.

Nunca $1-2 \quad 2 \quad-3 \quad-4$ Siempre

62. Utilizaré la autopista o autovía siempre que pueda.

$$
\text { Siempre } 1 \quad-2 \quad-\quad 3 \quad-4 \quad 4 \quad \text { Nunca }
$$

63. Haré caso de las indicaciones de reducción de la velocidad cuando haya obras en la vía.

Siempre $1-2-3-4 \quad-\quad-4$ Nunca

H) Seguridad proveniente del tráfico.

64. Creo que cuando hay tráfico la conducción es mas animada y emocionante.

$\begin{array}{lllllll}\text { Nada } & 1 & - & 2 & - & 3 & -\end{array}$ Mucho

65. Utilizaré preferentemente las vías de una sola dirección: autopistas, autovías y calles de sentido único.

66. Cuando tenga prisa intentaré adelantar.

$$
\text { Siempre } 1-2-3-4 \quad \text { Nunca }
$$

Nunca $1-2-3 \quad-4$ Siempre

67. Creo que me mentalizaré antes de coger el turismo de los posibles atascos o cuando me encuentre me lo tomaré con tranquilidad.
Siempre
$1 \quad-\quad 2$
$2 \quad-3 \quad-4$
Nunca

68. El tráfico por ciudad me pone nervioso/a, por eso cuando se conduce por ciudad lo mejor es acabar el trayecto cuanto antes.

\section{$\begin{array}{lllllllll}\text { Totalmente en desacuerdo } & 1 & - & 2 & - & 3 & - & 4 & \text { Totalmente de acuerdo }\end{array}$}

69. Cuando me encuentre con atascos, después intentaré ganar el tiempo perdido yendo a más velocidad.

$$
\text { Nunca } 1-2 \quad-3-4 \quad \text { Siempre }
$$

70. Conozco los puntos negros de mi entorno próximo.

$$
\text { Todos } 1 \quad-2 \quad 2 \quad 3 \quad-4 \text { Ninguno }
$$

I) Seguridad proveniente de la climatología: lluvia, nieve, hielo, viento

71. Si nieva, lo mejor es elegir una ruta alternativa aunque sea más larga.

$$
\text { Siempre } 1 \quad-2 \quad 2 \quad 3-4 \quad-4 \text { Nunca }
$$

72. Creo que los turismos de hoy en día se adherirían bien si acelerase en una carretera helada.

\section{$\begin{array}{lllllllll}\text { Totalmente en desacuerdo } & 1 & - & 2 & - & 3 & - & 4 & \text { Totalmente de acuerdo }\end{array}$}

73. Con un turismo con antibloqueo iría más confiado/a los días de lluvia y nieve.

$$
\text { Nunca } 1 \quad-2 \quad 2 \quad 3-4 \quad \text { Siempre }
$$

74. Revisaré y prepararé el turismo cuando tenga que conducir por la nieve o para realizar trayectos largos.

$$
\text { Siempre } 1-2 \quad 2-3-4 \quad \text { Nunca }
$$

75. Cuando encuentre niebla, pondré las luces de cruce, reduciré la velocidad y no adelantaré.

76. Iré a la misma velocidad tanto si llueve como si no.

$$
\text { Siempre } 1-2 \quad-\quad 3 \quad-4 \quad \text { Nunca }
$$

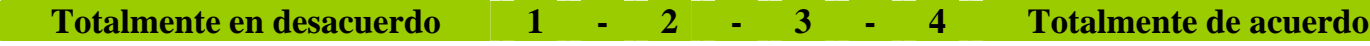

77. Los golpes de viento no se pueden prever. Lo que se debe hacer es controlar el vehículo cuando los recibes.

Totalmente en desacuerdo $1-2-2-3-4$ Totalmente de acuerdo

J) Seguridad proveniente de las normas y señales de tráfico. 
78. Cuando el semáforo empiece a ponerse rojo me daré prisa en pasar.

$$
\text { Nunca } 1-2-3-4 \text { Siempre }
$$

79. No es estrictamente necesaria detenerse en un stop. A veces se puede mirar y si no viene un turismo se puede pasar sin detenerse.

Totalmente en desacuerdo $1-2-3-4$ Totalmente de acuerdo

80. Cuando me encuentre con una señal que me de preferencia de paso obligaré a los otros conductores que no tienen preferencia que me dejen pasar.

$$
\begin{array}{llllllll}
\text { Nunca } & 1 & - & 2 & - & 3 & - & 4
\end{array} \text { Siempre }
$$

81. Hay muchas señales de tráfico que no sirven para nada.
Totalmente en desacuerdo

K) Test de madurez de la conducción eficaz.

\begin{tabular}{|c|}
\hline Más de 16 \\
\hline
\end{tabular}

82. ¿Cuántas prácticas he realizado?

83. Me equivoco cuando he de utilizar el limpiaparabrisas.

84. Me olvido de la marcha que llevo y la he de localizar con la mano.

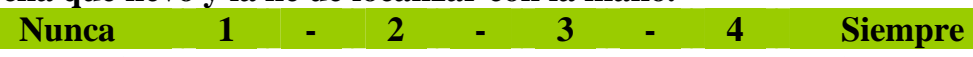

85. Tengo problemas para localizar los mandos de las luces cuando estoy conduciendo.

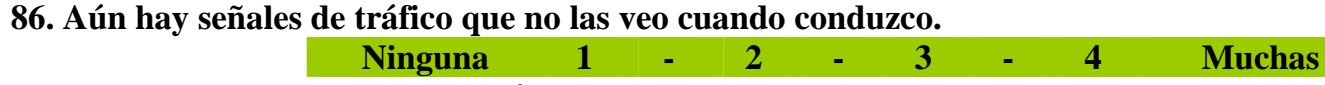

87. Cuando tengo que dejar una vía me cuesta encontrar el camino adecuado.

$$
\text { Nunca } 1-5-2-4 \text { Siempre }
$$

88. Mi nivel de hábitos de seguridad conduciendo es:

$$
\begin{array}{llllllll}
\text { Alto } & 1 & - & 2 & - & 3 & - & 4
\end{array} \text { Bajo }
$$

89. Cuando conduzco salgo de las líneas del carril por el que quiero ir.

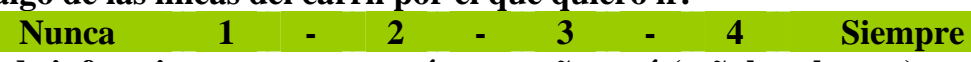

90. Creo que el numero de infracciones que cometeré en un año será (señala solo una):

$\begin{array}{llllllll}1 & \text { Cero o una } & 2 & \text { Dos } & 3 & \text { Tres } & 4 & \text { Cuatro y más }\end{array}$

\section{ANEXO 2}

\section{CUESTIONARIO ESPECÍFICO DE VELOCIDAD}

1.- Me gusta correr.

Totalmente en desacuerdo $1 \quad \begin{array}{llllllll} & - & - & - & - & \end{array} \quad$ Totalmente de acuerdo

2.- Creo que iré a $15 \mathrm{Km}$./h. más de la velocidad señalada.
Totalmente en desacuerdo 1 -

3.- Si puedo, por ciudad iré a más de $50 \mathrm{Km} . / \mathrm{h}$.

$\begin{array}{lllllllll}\text { Totalmente en desacuerdo } & 1 & - & - & - & - & \end{array} \quad$ Totalmente de acuerdo

4.- Creo que tengo habilidades para correr con facilidad.

Totalmente en desacuerdo $1-2-2-3-4 \quad$ Totalmente de acuerdo

5.- Pienso que correré más en las vías conocidas.

Totalmente en desacuerdo $\quad \begin{array}{llllllll} & - & 2 & - & 3 & - & 4 & \text { Totalmente de acuerdo }\end{array}$

6.- Creo que en autopista y otras vías rápidas me resultará sencillo correr con facilidad.

Totalmente en desacuerdo $1-2-2 \quad-\quad 3-4 \quad$ Totalmente de acuerdo

7.- Para mí, uno de los mejores atractivos de la conducción es la velocidad.

$\begin{array}{lllllll}\text { Totalmente en desacuerdo } & 1 & - & 2 & - & 3 & -\end{array} \quad$ Totalmente de acuerdo

8.- Pienso que los turismos actuales son para correr, por eso tienen las prestaciones que tienen.

$\begin{array}{lllllllll}\text { Totalmente en desacuerdo } & 1 & - & 2 & - & 3 & - & 4 & \text { Totalmente de acuerdo }\end{array}$

9.- Si aprendo a correr me ayudará a vivir mejor.

$\begin{array}{lllllllll}\text { Totalmente en desacuerdo } & 1 & - & - & - & - & \text { Totalmente de acuerdo }\end{array}$

10.- Cuando voy de acompañante, me gusta que el conductor corra.

Totalmente en desacuerdo $1-2-3-4$ Totalmente de acuerdo

11.- Pienso que un buen conductor o conductora debe saber correr.

Totalmente en desacuerdo $1-2-3-4$ Totalmente de acuerdo

12.- Si me esfuerzo en conducir bien podré correr más.

$\begin{array}{lllllllll}\text { Totalmente en desacuerdo } & 1 & - & 2 & - & 3 & - & 4 & \text { Totalmente de acuerdo }\end{array}$ 


\section{$\underline{\text { ABOUT THE AUTHORS / SOBRE LOS AUTORES }}$}

Jariot Garcia, Mercè (Merce.Jariot@uab.cat). Es profesora del Área MIDE del Departamento de Pedagogía Aplicada de la UAB. Sus principales líneas de trabajo están relacionadas con la evaluación de programas, la investigación e intervención en educación y seguridad vial y la orientación profesional - ocupacional. Es investigadora de la Cátedra de Formación y Educación Vial de la UAB y del Servei Català de Trànsit, directora del Equipo de Investigación en Educación y Seguridad Vial (ERESv) de la UAB y miembro del Equipo de Investigación en Infancia y Adolescencia en Riesgo Social (IARS) de la UAB. Buscar otros artículos de este autor en Scholar Google

Montané, Josep (Josep.Montane@uab.cat). Es Catedrático en la Universidad Autónoma de Barcelona. Sus principales líneas de trabajo están relacionadas con la orientación profesional y la prevención de accidentes de tráfico. Actualmente es el director de la "Cátedra de Formació i Educació Viàries” del "Servei Cátala de Trànsit y la UAB. Buscar otros artículos de este autor en Scholar Google Google: 


\title{
ARTICLE RECORD / FICHA DEL ARTÍCULO
}

\begin{tabular}{|c|c|}
\hline $\begin{array}{l}\text { Reference / } \\
\text { Referencia }\end{array}$ & $\begin{array}{l}\text { Jariot Garcia, Mercè \& Montané Capdevila, Josep (2009). Actitudes y velocidad en jóvenes. Aplicación de } \\
\text { un programa de educación vial. RELIEVE, v. 15, n. } 1 . \\
\text { http://www.uv.es/RELIEVE/v15n1/RELIEVEv15n1_2.htm. Consultado en (poner fecha). }\end{array}$ \\
\hline $\begin{array}{l}\text { Title / } \\
\text { Título }\end{array}$ & $\begin{array}{l}\text { Actitudes y velocidad en jóvenes. Aplicación de un programa de educación vial. [Attitude and speed in } \\
\text { young people. Implementation of a program of driver education] }\end{array}$ \\
\hline $\begin{array}{l}\text { Authors / } \\
\text { Autores }\end{array}$ & Jariot Garcia, Mercè \& Montané Capdevila, Josep \\
\hline $\begin{array}{l}\text { Review / } \\
\text { Revista }\end{array}$ & Revista ELectrónica de Investigación y EValuación Educativa (RELIEVE), v. 15, n. 1 \\
\hline ISSN & $1134-4032$ \\
\hline $\begin{array}{l}\text { Publication } \\
\text { date / } \\
\text { Fecha de } \\
\text { publicación }\end{array}$ & 2008 (Reception Date: 2009 April 14; Approval Date: 2009 March 8; Publication Date: 2009 March 2). \\
\hline $\begin{array}{l}\text { Abstract / } \\
\text { Resumen }\end{array}$ & $\begin{array}{l}\text { Contemporary Theories of change of attitudes defends that these depends on the way to think, to act and to } \\
\text { feel of the people. If we touch upon each one of these three main components related between we can influ- } \\
\text { ence in the predisposition to act. The application of a program of speed self-control to ample of young driv- } \\
\text { ers, using a design pretest-postest with control group and using a Factor Analysis shows that is possible the } \\
\text { change of attitudes. } \\
\text { Las teorías contemporáneas del cambio de actitudes defienden que éstas dependen de la manera de pensar, } \\
\text { actuar y sentir de las personas. Si se incide en cada uno de estos tres factores íntimamente relacionados } \\
\text { entre si podemos influir en la predisposición a actuar. La aplicación de un programa de autocontrol de ve- } \\
\text { locidad a una muestra de preconductores, utilizando un diseño pretest-postest con grupo control y utilizan- } \\
\text { do un análisis factorial pone de manifiesto que es posible el cambio de actitudes en los jóvenes. }\end{array}$ \\
\hline $\begin{array}{l}\text { Keywords / } \\
\text { Descriptores }\end{array}$ & $\begin{array}{l}\text { Change of attitudes; Speed; Investigation in road education and security; Road education and formation; } \\
\text { Road security. } \\
\text { Cambio de actitudes; Velocidad; Investigación en educación y seguridad vial; Educación y formación } \\
\text { viales; Seguridad vial. }\end{array}$ \\
\hline $\begin{array}{l}\text { Institution / } \\
\text { Institución }\end{array}$ & Universidad Autónoma de Barcelona (España). \\
\hline $\begin{array}{l}\text { Publication } \\
\text { site / } \\
\text { Dirección }\end{array}$ & http://www.uv.es/RELIEVE \\
\hline $\begin{array}{l}\text { Language / } \\
\text { Idioma }\end{array}$ & Español (Title, abstract and keywords in English ) \\
\hline
\end{tabular}

\section{Revista ELectrónica de Investigación y EValuación Educativa (RELIEVE)}

[ ISSN: 1134-4032 ]

\begin{abstract}
(C) Copyright, RELIEVE. Reproduction and distribution of this articles it is authorized if the content is no modified and their origin is indicated (RELIEVE Journal, volume, number and electronic address of the document).

(C) Copyright, RELIEVE. Se autoriza la reproducción y distribución de este artículo siempre que no se modifique el contenido y se indique su origen (RELIEVE, volumen, número y dirección electrónica del documento).
\end{abstract}

